\title{
EIV-Based Interference Alignment Scheme with CSI Uncertainties
}

\author{
Zhengmin Kong, ${ }^{1}$ Shixin Peng, ${ }^{2}$ Yuxuan Zhang, ${ }^{3}$ and Liang Zhong ${ }^{1}$ \\ ${ }^{1}$ Department of Automation, Wuhan University, Wuhan 430072, China \\ ${ }^{2}$ The State Grid Information and Communication Company of Hunan Electric Power Corp., Changsha 410007, China \\ ${ }^{3}$ Department of Electrical Engineering, Columbia University, New York, NY 10027, USA
}

Correspondence should be addressed to Shixin Peng; psx6050@163.com

Received 26 March 2015; Accepted 23 June 2015

Academic Editor: William Guo

Copyright (C) 2015 Zhengmin Kong et al. This is an open access article distributed under the Creative Commons Attribution License, which permits unrestricted use, distribution, and reproduction in any medium, provided the original work is properly cited.

A novel interference alignment (IA) scheme based on the errors-in-variables (EIV) mathematic model has been proposed to overcome the channel state information (CSI) estimation error for the MIMO interference channels. By solving an equivalently unconstrained optimization problem, the proposed IA scheme employing a weighted total least squares (WTLS) algorithm can obtain the solution to a constrained optimization problem for transmit precoding (TPC) matrices and minimizes the distortion caused by imperfect CSI according to the EIV model. It is shown that the design of TPC matrices can be realized through an efficient iterative algorithm. The convergence of the proposed scheme is presented as well. Simulation results show that the proposed IA scheme is robust over MIMO interference channels with imperfect CSI, which yields significantly better sum rate performance than the existing IA schemes such as distributed iterative IA, maximum signal-to-interference-plus-noise ratio (Max SINR), and minimum mean square error (MMSE) schemes.

\section{Introduction}

The importance of the interference management has been emphasized in $[1,2]$. It is of paramount importance to conceive efficient interference management schemes for multiuser wireless networks. Interference alignment (IA) has been proposed as a powerful and promising technique to mitigate the interference in multiuser interference channels [1-3]. In [1], Jafar and Shamai first characterized the degrees of freedom (DoF) for two-user multiple input multiple output (MIMO) interference channels. The earlier studies [1-3] of his group were focused on the DoF for various distributed systems. Since then, IA technique has been used to structure interfering signals to occupy a reduced-dimensional interference subspace at the receivers and thus maximize the multiplexing gain (or the sum rate of system).

Recently, various algorithms for IA and IA-inspired schemes in different scenarios [4-8] have been proposed. Among them, maximum signal-to-interference-plus-noise ratio (Max SINR) scheme [5] so far has been regarded as one of the most effective schemes, without being proven to be convergent. Some other kinds of iterative schemes, such as iterative CJ08 scheme [5] and MMSE scheme [9], are also widely used in IA. And most of these former works rely upon perfect channel state information (CSI). However, realistic practical channel estimation (CE), feedback, quantization, and so forth have errors. Therefore, CSI at the transmitter and receiver is far from being perfect. It is revealed in [10] that the performance of IA scheme is sensitively degraded due to the uncertainties of CSI. Lately, several works on IA schemes with imperfect channel knowledge have focused on this aspect. In [11], the authors investigate an iterative beamforming design algorithm so as to maximize the sum outage rate for MIMO interference channels. Specifically, in $[12,13]$, the authors propose a minimum mean squared error (MMSE) scheme that takes CSI error into account to align the interference. This MMSE approach is further extended to the robust MSE-based iterative transceiver designs in [14], which effectively improve the BER performance of MIMO interference system. A robust lattice alignment method designed by using the existing conventional Iterative IA algorithm is presented in [15] for quasistatic MIMO interference channels 
with imperfect CSI as well. In addition, [16, 17] investigate the effect of CSI mismatch and develop several adaptive interference alignment schemes with CSI error.

In contrast to previous works above, we, in this paper, take the errors-in-variables (EIV) model into consideration from the essence of IA with imperfect CSI and employ an unbiased parameter estimation technique in such a linear measurement error model instead of the conventional method of minimizing projector distances of interference subspaces. Hence, the estimation problem can be easily converted into the problem of solving a linear system of equations. Our goal is to overcome the distortion caused by the imperfect CSI at transmitters and optimize the sum rate performance under a given and feasible DoF. To this end, an EIV-based system model of interference management is established. Meanwhile, a weighted total least squares- (WTLS-) based estimation algorithm is proposed to update the transmit precoding (TPC) matrices for the implementation of accurate IA. Unlike the Max SINR scheme, our proposed WTLSbased (or EIV-based) IA scheme is proven to be convergent. Simulation results show that the proposed WTLS-based IA scheme can improve IA performance under the scenarios with different variances of error for direct link estimation and interference links estimations. Against this background, the major contributions of this paper are summarized as follows:

(1) We firstly introduce the EIV model into IA. More explicitly, we set out to establish an EIV-based system model of an IA scheme with imperfect CSI for the sake of exploiting the unbiased WTLS parameter estimation algorithm to estimate TPC matrix in such a linear measurement error model.

(2) We propose a WTLS-based IA scheme to convert the IA-based constrained optimization problem under the condition of imperfect CSI into an equivalently unconstrained optimization problem in the transmitted signal estimation. More explicitly, the unconstrained optimization problem is derived by minimizing analytically over the correction of the measurement data matrix, and an iterative algorithm for the solution of the unconstrained optimization problem is presented. What is more, our WTLS-based IA scheme is proven to be convergent by employing the large data matrix.

The rest of this paper is organized as follows. In the next section, the EIV-based system model is first introduced. In Section 3, we present the proposed WTLS-based IA scheme with CSI uncertainties and discuss the convergence issues. In Section 4, we demonstrate the proposed algorithm with numerical simulations. At last, we conclude with Section 5.

Notation. The superscript $*$, superscript $T$, and superscript $H$ stand, respectively, for conjugate, transpose, and Hermitian transpose. Matrices and vectors are set in bold-face letters. $\mathbf{A}(i,:)$ or $\mathbf{A}_{i,:}$ denotes the $i$ th row of $\mathbf{A}$, and $\mathbf{A}(:, j)$ or $\mathbf{A}_{:, j}$ denotes the $j$ th column of $\mathbf{A}$. I is the identity matrix and $\mathbf{0}$ is the all-zero matrix. $\mathbf{a}_{i, j}$ is the $(i, j)$ th element of $\mathbf{A}$. $\operatorname{cov}(\mathbf{x})$ denotes the covariance matrices of a random vector $\mathbf{x}$. $\|\mathbf{A}\|_{2}, E\{\mathbf{A}\}$, and $\operatorname{det}(\mathbf{A})$ denote $l_{2}$-norm, the expectation, and

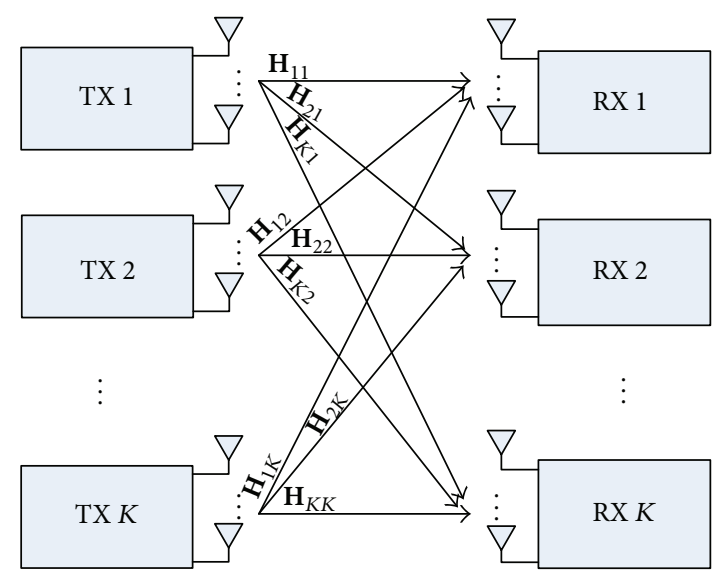

FIgURE 1: $K$-user MIMO interference channels model.

the determinant of $\mathbf{A}$, respectively. $\mathbb{C}^{M \times N}$ is the space of complex $M \times N$ matrices. $\mathscr{C} \mathscr{N}(\mathbf{a}, \mathbf{A})$ is complex Gaussian distribution with mean a and covariance matrix $\mathbf{A}$.

\section{EIV-Based System Model}

In this paper, we consider $K$-user IA scheme over MIMO interference channels. As shown in Figure 1, each transmitter and receiver is equipped with $N_{t}$ and $N_{r}$ antennas, respectively. The channel output at receiver $k$ is defined as follows:

$$
\mathbf{y}_{k}=\sum_{l=1}^{K} \mathbf{H}_{k l} \mathbf{x}_{l}+\mathbf{z}_{k}=\mathbf{H}_{k k} \mathbf{V}_{k} \mathbf{s}_{k}+\sum_{l \neq k}^{K} \mathbf{H}_{k l} \mathbf{V}_{l} \mathbf{s}_{l}+\mathbf{z}_{k}
$$

where $\mathbf{y}_{k} \in \mathbb{C}^{N_{r} \times 1}$ represents the received signal vector at receiver $k$ and $\mathbf{x}_{l} \in \mathbb{C}^{N_{t} \times 1}$ is the $N_{t} \times 1$ transmitted signal vector at transmitter $l . \mathbf{H}_{k l} \in \mathbb{C}^{N_{r} \times N_{t}}$ represents the channel fade coefficients from transmitter $l$ to receiver $k$ for $l, k=1, \ldots, K$, where each element is assumed to obey an independent and identically distributed (i.i.d.) complex Gaussian random process with zero mean and unit variance $\mathscr{C} \mathcal{N}(0,1) . \mathbf{z}_{k} \in \mathbb{C}^{N_{r} \times 1}$ is noise vector at receiver $k$, which is complex-valued additive white gaussian noise (AWGN) with elements normal distributed as $\mathscr{C} \mathcal{N}\left(\mathbf{0}, \sigma^{2} \mathbf{I}\right) . \mathbf{V}_{k} \in \mathbb{C}^{N_{t} \times d_{k}}$ is the TPC matrix with unit-norm and linearly independent columns at transmitter $k$, where $d_{k}$ is the DoF to meet feasibility of IA scheme at transmitter $k$. $s_{k}$ represents the data streams from transmitter $k$, where the transmitted streams are i.i.d. such that $E\left\{\mathbf{s}_{k} \mathbf{s}_{k}^{H}\right\}=\mathbf{I}$.

Furthermore, the data streams received at receiver $k$ can be described as

$$
\widehat{\mathbf{s}}_{k}=\mathbf{U}_{k}^{H} \mathbf{y}_{k}=\mathbf{U}_{k}^{H} \sum_{l=1}^{K} \mathbf{H}_{k l} \mathbf{V}_{l} \mathbf{s}_{l}+\mathbf{U}_{k}^{H} \mathbf{z}_{k},
$$

where $\mathbf{U}_{k} \in \mathbb{C}^{N_{r} \times d_{k}}$ is receiving filter matrix with unit-norm and linearly independent columns at receiver $k$.

In most IA schemes, perfect CSI is assumed. However, CSI is far from being perfect in realistic practical system. We assume that only imperfect estimation of global CSI is 
available at each terminal. The feedback channels are assumed to be error-free. The imperfect CSI model can be quantized according to

$$
\mathbf{H}_{k l}=\widehat{\mathbf{H}}_{k l}+\widetilde{\mathbf{H}}_{k l}, \quad \forall l, k \in\{1,2, \ldots, K\},
$$

where $\widehat{\mathbf{H}}_{k l}$ is the estimated channel matrix from transmitter $l$ to receiver $k$ and $\widetilde{\mathbf{H}}_{k l}$ is the CSI error matrix between the true and available information. This error matrix is assumed to be i.i.d. zero-mean complex Gaussian, and

$$
\begin{aligned}
E\left\{\widetilde{\mathbf{H}}_{k k} \widetilde{\mathbf{H}}_{k k}^{H}\right\} & =\sigma_{h, \mathrm{I}}^{2} \mathbf{I}, \quad \forall k \in\{1,2, \ldots, K\}, \\
E\left\{\widetilde{\mathbf{H}}_{k l} \widetilde{\mathbf{H}}_{k l}^{H}\right\} & =\sigma_{h, \mathrm{II}}^{2} \mathbf{I}, \quad \forall l \neq k \in\{1,2, \ldots, K\}, \\
E\left\{\widetilde{\mathbf{H}}_{k l} \widetilde{\mathbf{H}}_{i j}^{H}\right\} & =\mathbf{0}, \quad \forall k \neq i, \quad l \neq j,
\end{aligned}
$$

where the parameters $\sigma_{h, \mathrm{I}}^{2}$ and $\sigma_{h, \mathrm{II}}^{2}$ indicate the variance of error for direct link estimation and interference links estimations [10], respectively. From (3) to (6), we know $\widetilde{\mathbf{H}}_{k k} \sim \mathscr{C} \mathscr{N}\left(0, \mathbf{I}-\sigma_{h, \mathrm{I}}^{2} \mathbf{I}\right)$ and $\widetilde{\mathbf{H}}_{k l} \sim \mathscr{C} \mathscr{N}\left(0, \mathbf{I}-\sigma_{h, \mathrm{II}}^{2} \mathbf{I}\right)$. If $\sigma_{h, \mathrm{I}}^{2}, \sigma_{h, \mathrm{II}}^{2} \rightarrow 0$, then the imperfect CSI model reduces to the case that perfect CSI is available at terminals, and when $\sigma_{h, \mathrm{I}}^{2}$, $\sigma_{h, \mathrm{II}}^{2} \rightarrow 1$, it represents that no CSI is available terminals. The parameters $\sigma_{h, \mathrm{I}}^{2}$ and $\sigma_{h, \mathrm{II}}^{2}$ can potentially have different values corresponding to different accuracy of channel estimation of the direct links and that of the interference links.

Due to imperfect CSI, then (2) can be written as

$$
\begin{aligned}
\widehat{\mathbf{s}}_{k}= & \mathbf{U}_{k}^{H} \sum_{i=1}^{K} \widehat{\mathbf{H}}_{k i} \mathbf{V}_{i} \mathbf{s}_{i}+\mathbf{U}_{k}^{H} \mathbf{z}_{k} \\
= & \mathbf{U}_{k}^{H} \widehat{\mathbf{H}}_{k k} \mathbf{V}_{k} \mathbf{s}_{k}+\mathbf{U}_{k}^{H} \sum_{l \neq k}^{K} \widehat{\mathbf{H}}_{k l} \mathbf{V}_{l} \mathbf{s}_{l}+\mathbf{U}_{k}^{H} \mathbf{z}_{k} \\
= & \mathbf{U}_{k}^{H}\left(\mathbf{H}_{k k}-\widetilde{\mathbf{H}}_{k k}\right) \mathbf{V}_{k} \mathbf{s}_{k} \\
& +\mathbf{U}_{k}^{H}\left(\sum_{l \neq k}^{K} \mathbf{H}_{k l} \mathbf{V}_{l} \mathbf{s}_{l}-\sum_{l \neq k}^{K} \widetilde{\mathbf{H}}_{k l} \mathbf{V}_{l} \mathbf{s}_{l}\right)+\mathbf{U}_{k}^{H} \mathbf{z}_{k} .
\end{aligned}
$$

From (7), we hardly find that the IA scheme with CSI error is relative with EIV model. However, when the last two terms on the right-hand part of (7) are moved to the left-hand part, it will be clear that

$$
\begin{aligned}
\widehat{\mathbf{s}}_{k}+ & {\left[-\mathbf{U}_{k}^{H}\left(\sum_{l \neq k}^{K} \mathbf{H}_{k l} \mathbf{V}_{l} \mathbf{s}_{l}-\sum_{l \neq k}^{K} \widetilde{\mathbf{H}}_{k l} \mathbf{V}_{l} \mathbf{s}_{l}\right)-\mathbf{U}_{k}^{H} \mathbf{z}_{k}\right] } \\
& =\left(\mathbf{U}_{k}^{H} \mathbf{H}_{k k}-\mathbf{U}_{k}^{H} \widetilde{\mathbf{H}}_{k k}\right) \mathbf{V}_{k} \mathbf{s}_{k} .
\end{aligned}
$$

Obviously, from above (8), the terms of interference and noise $-\mathbf{U}_{k}^{H}\left(\sum_{l \neq k}^{K} \mathbf{H}_{k l}-\sum_{l \neq k}^{K} \widetilde{\mathbf{H}}_{k l}\right) \mathbf{V}_{l} \mathbf{s}_{l}-\mathbf{U}_{k}^{H} \mathbf{z}_{k}$ are considered as the measurement errors of the interested parameter $\widehat{\mathbf{s}}_{k}$, while the CSI error term $\mathbf{U}_{k}^{H} \widetilde{\mathbf{H}}_{k k}$ is considered as the measurement error of the interested parameter $\mathbf{U}_{k}^{H} \mathbf{H}_{k k}$. Definitely, this IA system model with imperfect CSI in (8) satisfies the EIV model $[18,19]$.
Briefly, the above EIV-based system model can be described by a corrected system of equations $[18,19] \mathbf{Y}+\Delta \mathbf{Y}=$ $(\mathbf{H}+\Delta \mathbf{H}) \mathbf{X}$, where $\mathbf{Y} \approx \mathbf{H X}$ and $\mathbf{X}$ is the parameter to be estimated, while $\Delta \mathbf{Y}$ and $\Delta \mathbf{H}$ are the perturbations of $\mathbf{Y}$ and $\mathbf{H}$, respectively.

As a result, the transmitted signal estimation problem for the IA with imperfect CSI can be defined as a constrained optimization problem: an appropriate cost function depending on the data is minimized over the estimated parameters.

The classical method, so-called generalized total least squares (TLS) $[18,20-22]$, is proposed as a parameter estimation technique for the EIV model when all elements in channel matrices are perturbed by i.i.d. Particularly, the WTLS [19, 23-25] became popular in the mathematics field during this decade since its estimator has a better statistical accuracy under more general noise assumptions. So in the next section we will focus our attention on the WTLS in a realistic CSI error-based interference channel.

\section{WTLS-Based Interference Alignment Scheme with Imperfect CSI}

In this section, the proposed WTLS-based IA scheme will be investigated to solve the IA-based constrained optimization under the condition of imperfect CSI. While minimizing analytically over the correction matrix, the constrained optimization problem can be changed into an equivalently unconstrained optimization problem. And an effective way to set the weights will also be considered. At the end of this section, an iterative algorithm will be presented for the sake of solving the WTLS-based optimization.

3.1. IA-Based Constrained Optimization with CSI Uncertainties. Looking back on (8), we assume that perfect IA is available and $\mathbf{V}_{k} \mathbf{s}_{k}$ is the true value of its approximation estimation $\widehat{\mathbf{V}}_{k} \mathbf{s}_{k}(k \in\{1,2, \ldots, K\})$ such that

$$
\begin{array}{r}
\widehat{\mathbf{s}}_{k}+\mathbf{U}_{k}^{H}\left(-\sum_{l \neq k}^{K} \widehat{\mathbf{H}}_{k l} \widehat{\mathbf{V}}_{l} \mathbf{s}_{l}-\mathbf{z}_{k}\right)=\mathbf{U}_{k}^{H} \widehat{\mathbf{H}}_{k k} \widehat{\mathbf{V}}_{k} \mathbf{s}_{k}, \\
\widehat{\mathbf{s}}_{k} \approx \mathbf{U}_{k}^{H} \mathbf{H}_{k k} \widehat{\mathbf{V}}_{k} \mathbf{s}_{k},
\end{array}
$$

where we start with arbitrary TPC matrix and receiving filter matrix. Here we define a measurement data matrix $\mathbf{D}$ with $\mathrm{CSI}$ errors at $k$ th receiver

$$
\begin{aligned}
\mathbf{D} & \triangleq\left[\begin{array}{ll}
\mathbf{U}_{k}^{H} \widehat{\mathbf{H}}_{k k} & \widehat{\mathbf{s}}_{k}-\mathbf{U}_{k}^{H}\left(\sum_{l \neq k}^{K} \widehat{\mathbf{H}}_{k l} \widehat{\mathbf{V}}_{l} \mathbf{s}_{l}+\mathbf{z}_{k}\right)
\end{array}\right] \\
& =\left[\begin{array}{ll}
\mathbf{U}_{k}^{H} \widehat{\mathbf{H}}_{k k} & \mathbf{U}_{k}^{H} \widehat{\mathbf{H}}_{k k} \widehat{\mathbf{V}}_{k} \mathbf{s}_{k}
\end{array}\right]=\left[\begin{array}{ll}
\mathbf{A} & \mathbf{B}
\end{array}\right],
\end{aligned}
$$

where $\mathbf{A} \triangleq \mathbf{U}_{k}^{H} \widehat{\mathbf{H}}_{k k}$ and $\mathbf{B} \triangleq \mathbf{U}_{k}^{H} \widehat{\mathbf{H}}_{k k} \widehat{\mathbf{V}}_{k} \mathbf{s}_{k}$. According to (3), the measurement data matrix $\mathbf{D}$ can be given by

D

$$
=\left[\mathbf{U}_{k}^{H} \mathbf{H}_{k k}-\mathbf{U}_{k}^{H} \widetilde{\mathbf{H}}_{k k} \widehat{\mathbf{s}}_{k}-\mathbf{U}_{k}^{H}\left(\sum_{l \neq k}^{K} \widehat{\mathbf{H}}_{k l} \widehat{\mathbf{V}}_{l} \mathbf{s}_{l}+\mathbf{z}_{k}\right)\right]
$$




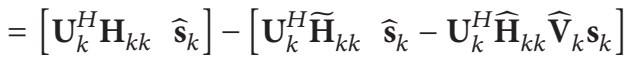

$$
\begin{aligned}
& =\mathbf{D}_{\text {tru }}-\left[\begin{array}{ll}
\widetilde{\mathbf{A}} & \widetilde{\mathbf{B}}
\end{array}\right]=\mathbf{D}_{\text {tru }}-\widetilde{\mathbf{D}} \text {, }
\end{aligned}
$$

where $\mathbf{D}_{\text {tru }} \triangleq\left[\begin{array}{ll}\mathbf{U}_{k}^{H} \mathbf{H}_{k k} & \widehat{\mathbf{s}}_{k}\end{array}\right], \widetilde{\mathbf{A}} \triangleq \mathbf{U}_{k}^{H} \widetilde{\mathbf{H}}_{k k}, \widetilde{\mathbf{B}} \triangleq \widehat{\mathbf{s}}_{k}-$ $\mathbf{U}_{k}^{H} \widehat{\mathbf{H}}_{k k} \widehat{\mathbf{V}}_{k} \mathbf{s}_{k}$, and $\widetilde{\mathbf{D}} \triangleq\left[\begin{array}{ll}\widetilde{\mathbf{A}} & \widetilde{\mathbf{B}}\end{array}\right] . \mathbf{D}_{\text {tru }}$ is the true value of data matrix $\mathbf{D}$, and $\widetilde{\mathbf{D}}$ is measurement error. And $\widetilde{\mathbf{d}}_{i}, i \in$ $\left\{1,2, \ldots, d_{k}\right\}$, is $i$ th independent column of $\widetilde{\mathbf{D}}^{H}$; it is with covariance matrices (covariance information of measurement error)

$$
\mathbf{W}_{i} \triangleq \operatorname{cov}\left(\widetilde{\mathbf{d}}_{i}\right), \quad i \in\left\{1,2, \ldots, d_{k}\right\} .
$$

We define an extended matrix of the approximate estimation $\widehat{\mathbf{V}}_{k} \mathbf{s}_{k}$

$$
\widehat{\mathbf{X}} \triangleq\left[\begin{array}{c}
\widehat{\mathbf{V}}_{k} \mathbf{s}_{k} \\
-\mathbf{I}
\end{array}\right] \triangleq\left[\begin{array}{c}
\mathbf{X} \\
-\mathbf{I}
\end{array}\right], \quad \forall k \in\{1,2, \ldots, K\}
$$

An alternative expression for the EIV-based system model (8) is

$$
\begin{aligned}
\mathbf{D} \widehat{X} & =\mathbf{0}, \\
\mathbf{D}_{\text {tru }} \mathbf{X}_{\text {tru }} & \approx 0,
\end{aligned}
$$

with

$$
\mathbf{X}_{\mathrm{tru}} \triangleq\left[\begin{array}{c}
\mathbf{V}_{k} \mathbf{s}_{k} \\
-\mathbf{I}
\end{array}\right] \triangleq\left[\begin{array}{c}
\mathbf{X}_{0} \\
-\mathbf{I}
\end{array}\right], \quad \forall k \in\{1,2, \ldots, K\}
$$

provided that the initial data matrix $\mathbf{D}$ is available and the error weights information is $\left\{\mathbf{W}_{i}\right\}_{i=1}^{d_{k}}$, corresponding to each row of data matrix $\mathbf{D}$, which totally depends on the quality of CSI. When the data matrix $\mathbf{D}$ is confirmed, we set out to seek for a minimal correction $\Delta \mathbf{D}$ to compensate for the measurement errors $\widetilde{\mathbf{D}}$, so that the WTLS optimization [23] in the presence of a mixture of perfect CSI and CSI error can be defined as

$$
\begin{aligned}
\min _{\widehat{\mathbf{V}}_{k} \mathbf{s}_{k}, \Delta \mathbf{D}} & \sum_{i=1}^{d_{k}}\left\|\left(\sqrt{\mathbf{W}_{i}}\right)^{-1} \Delta \mathbf{d}_{i}\right\|_{2}^{2}, \\
\text { s.t. } & (\mathbf{D}+\Delta \mathbf{D})\left[\begin{array}{c}
\widehat{\mathbf{V}}_{k} \mathbf{s}_{k} \\
-\mathbf{I}
\end{array}\right]=\mathbf{0},
\end{aligned}
$$

where $\Delta \mathbf{D}^{H}=\left[\Delta \mathbf{d}_{1}, \Delta \mathbf{d}_{2}, \ldots, \Delta \mathbf{d}_{d_{k}}\right]$. The optimization variables are $\mathbf{V}_{k} \mathbf{s}_{k}$ and $\Delta \mathbf{D}$. Let $\left(\widehat{\mathbf{V}}_{k, \text { opt }} \mathbf{s}_{k}, \Delta \mathbf{D}_{\text {opt }}\right)$ be an optimal point of the WTLS optimization problem. Thereupon, $\widehat{\mathbf{V}}_{k \text {,opt }} \mathbf{s}_{k}$ is an optimal WTLS estimation of the true value $\mathbf{V}_{k} \mathbf{s}_{k}$ and $\mathbf{D}+\Delta \mathbf{D}_{\text {opt }}$ is an optimal WTLS estimation of the true data $\mathrm{D}_{\text {tru }}$.
The first step of WTLS optimization is to minimize the cost function. The minimization with respect to the correction can be carried out as follows:

$$
\begin{aligned}
f(\mathbf{X})=\min _{\Delta \mathbf{d}_{1}, \Delta \mathbf{d}_{2}, \ldots, \Delta \mathbf{d}_{d_{k}}} & \sum_{i=1}^{d_{k}}\left\|\left(\sqrt{\mathbf{W}_{i}}\right)^{-1} \Delta \mathbf{d}_{i}\right\|_{2}^{2}, \\
\text { s.t. } & \mathbf{D} \widehat{\mathbf{X}}+\left[\begin{array}{c}
\Delta \mathbf{d}_{1}^{H} \widehat{\mathbf{X}} \\
\vdots \\
\Delta \mathbf{d}_{d_{k}}^{H} \widehat{\mathbf{X}}
\end{array}\right]=\mathbf{0 .}
\end{aligned}
$$

For a certain $\widehat{\mathbf{V}}_{k} \mathbf{s}_{k},(k \in\{1,2, \ldots, K\}), \widehat{\mathbf{X}}$ is fixed and the constraint of (17) can be treated as linear equations to optimize variables $\left\{\Delta \mathbf{d}_{i}\right\}_{i=1}^{d_{k}}$. The set of linear equations in $\left\{\Delta \mathbf{d}_{i}\right\}_{i=1}^{d_{k}}$ can be expressed as

$$
\begin{aligned}
\mathbf{D} \widehat{\mathbf{X}}+\left[\begin{array}{c}
\Delta \mathbf{d}_{1}^{H} \widehat{\mathbf{X}} \\
\vdots \\
\Delta \mathbf{d}_{d_{k}}^{H} \widehat{\mathbf{X}}
\end{array}\right]= & \mathbf{0} \\
& \Longleftrightarrow \widehat{\mathbf{X}}^{H} \Delta \mathbf{d}_{i}=-\left[(\mathbf{D} \widehat{\mathbf{X}})_{i,:}\right]^{H}, \\
& \quad i=1,2, \ldots, d_{k} .
\end{aligned}
$$

Let us define residual matrix $Q(\mathbf{X}) \triangleq \mathbf{D} \widehat{\mathbf{X}}$. The $i$ th row of the $Q(\mathbf{X})$ is denoted by $q_{i}^{H}(\mathbf{X})$; that is, $Q^{H}(\mathbf{X})=$ $\left[q_{1}(\mathbf{X}), q_{2}(\mathbf{X}), \ldots, q_{d_{k}}(\mathbf{X})\right]$. As a result, the constraint of (17) can be treated the same way as

$$
\widehat{\mathbf{X}}^{H} \Delta \mathbf{d}_{i}=-q_{i}(\mathbf{X}), \quad i=1,2, \ldots, d_{k},
$$

and thus the constrained optimization problem $f(\mathbf{X})$ can be separated according to different $\Delta \mathbf{d}_{i}$. In this way, the constrained minimization problem (17) is transformed to $d_{k}$ independent optimization problems

$$
\begin{aligned}
f(\mathbf{X})= & \sum_{i=1}^{d_{k}} f_{i}(\mathbf{X}), \\
f_{i}(\mathbf{X})=\min _{\Delta \mathbf{d}_{i}} & \left\|\left(\sqrt{\mathbf{W}_{i}}\right)^{-1} \Delta \mathbf{d}_{i}\right\|_{2}^{2}, \\
\text { s.t. } & \widehat{\mathbf{X}}^{H} \Delta \mathbf{d}_{i}=-q_{i}(\mathbf{X}), \\
& i=1,2, \ldots, d_{k},
\end{aligned}
$$

and the optimal correction is

$$
\Delta \mathbf{d}_{i, \mathrm{opt}}=-\mathbf{W}_{i} \widehat{\mathbf{X}}\left(\widehat{\mathbf{X}}^{H} \mathbf{W}_{i} \widehat{\mathbf{X}}\right)^{-1} q_{i}(\mathbf{X}), \quad i=1,2, \ldots, d_{k} .
$$

Employing (21) and the solution of $\Delta \mathbf{d}_{i}$ can be written as

$$
\Delta \mathbf{D}_{\mathrm{opt}}=\left[\begin{array}{c}
q_{1}^{H}(\mathbf{X})\left(\widehat{\mathbf{X}}^{H} \mathbf{W}_{1} \widehat{\mathbf{X}}\right)^{-1} \widehat{\mathbf{X}}^{H} \mathbf{W}_{1} \\
\vdots \\
q_{d_{k}}^{H}(\mathbf{X})\left(\widehat{\mathbf{X}}^{H} \mathbf{W}_{d_{k}} \widehat{\mathbf{X}}\right)^{-1} \widehat{\mathbf{X}}^{H} \mathbf{W}_{d_{k}}
\end{array}\right] .
$$


Hence, the solution of (17) becomes the well-known function $[18,26]$ as follows:

$$
\begin{aligned}
f(\mathbf{X}) & =\sum_{i=1}^{d_{k}} q_{i}^{H}(\mathbf{X})\left(\widehat{\mathbf{X}}^{H} \mathbf{W}_{i} \widehat{\mathbf{X}}\right)^{-1} q_{i}(\mathbf{X}) \\
& =\sum_{i=1}^{d_{k}} q_{i}^{H}(\mathbf{X}) R_{i}^{-1}(\mathbf{X}) q_{i}(\mathbf{X}),
\end{aligned}
$$

where $R_{i}(\mathbf{X}) \triangleq \widehat{\mathbf{X}}^{H} \mathbf{W}_{i} \widehat{\mathbf{X}}$.

As a result, the WTLS constrained optimization problem (17) is changed into an equivalently unconstrained optimization problem as follows:

$$
\min _{\mathbf{X}} f(\mathbf{X})
$$

3.2. Unconstrained Optimization. The second step of the WTLS optimization is to solve the unconstrained optimization problem (24). However, generally, the analytic solution is unavailable $[18,23]$. Thus, we propose a numerical method to solve the unconstrained optimization problem.

From the unconstrained optimization problem (24), we have the necessary condition for (23)

$$
\nabla_{\mathbf{x}^{*}} f(\mathbf{X})=\mathbf{0},
$$

where $\nabla_{\mathbf{x}^{*}} f(\mathbf{X})$ is conjugate gradient [27] for the unconstrained optimization problem (24).

According to the matrix theory $[27,28]$, the conjugate gradient which is presented in the appendix is

$$
\begin{gathered}
\nabla_{\mathbf{x}^{*}} f(\mathbf{X})=\sum_{i=1}^{d_{k}}\left\{R _ { i } ^ { - 1 } ( \mathbf { X } ) q _ { i } ^ { * } ( \mathbf { X } ) \left[\mathbf{a}_{i}^{T}\right.\right. \\
-q_{i}(\mathbf{X}) R_{i}^{-1}(\mathbf{X}) \mathbf{X}^{T} \operatorname{cov}\left(\widetilde{\mathbf{a}}_{i}^{*}\right) \\
\left.\left.+q_{i}(\mathbf{X}) R_{i}^{-1}(\mathbf{X}) \operatorname{cov}\left(\widetilde{b}_{i}^{*}, \widetilde{\mathbf{a}}_{i}^{*}\right)\right]\right\},
\end{gathered}
$$

where $\mathbf{a}_{i}, \widetilde{\mathbf{a}}_{i}, b_{i}$, and $\widetilde{b}_{i}$ are, respectively, the $i$ th column of $\mathbf{A}^{H}$, $\widetilde{\mathbf{A}}^{H}, \mathbf{B}^{H}$, and $\widetilde{\mathbf{B}}^{H}$. Note that, in the EIV-based IA system, $\mathbf{s}_{k}$ and $\widehat{\mathbf{X}}$ are vectors; thus the solution of $\mathbf{X}$ is univariate case. In this case, $R_{i}(\mathbf{X})$ is a single component matrix, while $q_{i}(\mathbf{X}), b_{i}$, and $\widetilde{b}_{i}$ are all single component vectors. The data weights are

$$
\begin{aligned}
\mathbf{W}_{i} & =\operatorname{cov}\left(\widetilde{\mathbf{d}}_{i}\right)=\left[\begin{array}{cc}
\operatorname{cov}\left(\widetilde{\mathbf{a}}_{i}\right) & \operatorname{cov}\left(\widetilde{\mathbf{a}}_{i}, \widetilde{b}_{i}\right) \\
\operatorname{cov}\left(\widetilde{b}_{i}, \widetilde{\mathbf{a}}_{i}\right) & \operatorname{cov}\left(\widetilde{b}_{i}\right)
\end{array}\right] \\
& \triangleq\left[\begin{array}{cc}
\mathbf{w}_{\widetilde{\mathbf{a}}_{i}} & \mathbf{w}_{\widetilde{\mathbf{a}}_{i} \widetilde{b}_{i}} \\
\mathbf{w}_{\tilde{b}_{i} \widetilde{\mathbf{a}}_{i}} & \mathbf{w}_{\tilde{b}_{i}}
\end{array}\right], \quad i=1,2, \ldots, d_{k} .
\end{aligned}
$$

Since there is no closed-form solution for (25), we use an iterative algorithm to solve the problem. Let $\mathbf{X}^{(l)}$ represent the approximate value of the $l$ th iterative result and let $\mathbf{X}^{(l+1)}$ represent the value of the $l+1$ th iterative result. Thus, we define the approximation function of (24) as follows:

$$
\begin{aligned}
& G\left(\mathbf{X}^{(l+1)}, \mathbf{X}^{(l)}\right)=\sum_{i=1}^{d_{k}}\left\{R_{i}^{-1}\left(\mathbf{X}^{(l)}\right) q_{i}^{*}\left(\mathbf{X}^{(l)}\right) q_{i}\left(\mathbf{X}^{(l)}\right)\right. \\
& \cdot R_{i}^{-1}\left(\mathbf{X}^{(l)}\right)\left[\mathbf{w}_{\tilde{b}_{i}^{*} \tilde{\mathbf{a}}_{i}^{*}}-\left(\mathbf{X}^{(l+1)}\right)^{T} \mathbf{w}_{\tilde{\mathbf{a}}_{i}^{*}}\right]+R_{i}^{-1}\left(\mathbf{X}^{(l)}\right) \\
& \left.\cdot q_{i}^{*}\left(\mathbf{X}^{(l+1)}\right) \mathbf{a}_{i}^{T}\right\} .
\end{aligned}
$$

Substituting the expression of $q_{i}(\mathbf{X})$ (the expression of $q_{i}(\mathbf{X})$ is detailed in the appendix) into (28), thus (28) can be rewritten as

$$
\begin{aligned}
G & \left(\mathbf{X}^{(l+1)}, \mathbf{X}^{(l)}\right)=\sum_{i=1}^{d_{k}}\left\{R_{i}^{-1}\left(\mathbf{X}^{(l)}\right)\left[\left(\mathbf{X}^{(l+1)}\right)^{T} \mathbf{a}_{i}^{*}-b_{i}^{*}\right]\right. \\
\cdot & \mathbf{a}_{i}^{T}+R_{i}^{-1}\left(\mathbf{X}^{(l)}\right)\left[\left(\mathbf{X}^{(l)}\right)^{T} \mathbf{a}_{i}^{*}-b_{i}^{*}\right] \\
\cdot & {\left[\left(\mathbf{X}^{(l)}\right)^{H} \mathbf{a}_{i}-b_{i}\right] R_{i}^{-1}\left(\mathbf{X}^{(l)}\right) } \\
\cdot & {\left.\left[\mathbf{w}_{\tilde{b}_{i}^{*} \mathbf{a}_{i}^{*}}-\left(\mathbf{X}^{(l+1)}\right)^{T} \mathbf{w}_{\widetilde{\mathbf{a}}_{i}^{*}}\right]\right\} . }
\end{aligned}
$$

Hence, the approximation $\mathbf{X}^{(l+1)}$ is defined as the solution of the following equation:

$$
G\left(\mathbf{X}^{(l+1)}, \mathbf{X}^{(l)}\right)=0 .
$$

Substituting (29) into (30), we obtain a system of linear equations with respect to $\mathbf{X}^{(l+1)}$,

$$
\begin{aligned}
& \sum_{i=1}^{d_{k}}\left\{R_{i}^{-1}\left(\mathbf{X}^{(l)}\right)\left[\left(\mathbf{X}^{(l)}\right)^{T} \mathbf{a}_{i}^{*}-b_{i}^{*}\right]\left[\left(\mathbf{X}^{(l)}\right)^{H} \mathbf{a}_{i}-b_{i}\right]\right. \\
& \cdot R_{i}^{-1}\left(\mathbf{X}^{(l)}\right)\left(\mathbf{X}^{(l+1)}\right)^{T} \mathbf{w}_{\tilde{\mathbf{a}}_{i}^{*}}-R_{i}^{-1}\left(\mathbf{X}^{(l)}\right)\left(\mathbf{X}^{(l+1)}\right)^{T} \\
& \left.\cdot \mathbf{a}_{i}^{*} \mathbf{a}_{i}^{T}\right\}=\sum_{i=1}^{d_{k}}\left\{R_{i}^{-1}\left(\mathbf{X}^{(l)}\right)\left[\left(\mathbf{X}^{(l)}\right)^{T} \mathbf{a}_{i}^{*}-b_{i}^{*}\right]\right. \\
& \cdot\left[\left(\mathbf{X}^{(l)}\right)^{H} \mathbf{a}_{i}-b_{i}\right] R_{i}^{-1}\left(\mathbf{X}^{(l)}\right) \mathbf{w}_{\tilde{b}_{i}^{*} \mathbf{a}_{i}^{*}}-R_{i}^{-1}\left(\mathbf{X}^{(l)}\right) \\
& \left.\cdot b_{i} \mathbf{a}_{i}^{T}\right\} \\
& \Longrightarrow \sum_{i=1}^{d_{k}}\left\{R_{i}^{-1}\left(\mathbf{X}^{(l)}\right)\left[\left(\mathbf{X}^{(l)}\right)^{T} \mathbf{a}_{i}^{*}-b_{i}^{*}\right]\left[\mathbf{a}_{i}^{T}\left(\mathbf{X}^{(l)}\right)^{*}-b_{i}\right]\right. \\
& \cdot R_{i}^{-1}\left(\mathbf{X}^{(l)}\right)\left(\mathbf{X}^{(l+1)}\right)^{T} \mathbf{w}_{\tilde{\mathbf{a}}_{i}^{*}}-R_{i}^{-1}\left(\mathbf{X}^{(l)}\right)\left(\mathbf{X}^{(l+1)}\right)^{T} \\
& \left.\cdot \mathbf{a}_{i}^{*} \mathbf{a}_{i}^{T}\right\}^{T}=\sum_{i=1}^{d_{k}}\left\{R_{i}^{-1}\left(\mathbf{X}^{(l)}\right)\left[\left(\mathbf{X}^{(l)}\right)^{T} \mathbf{a}_{i}^{*}-b_{i}^{*}\right]\right. \\
& \cdot\left[\mathbf{a}_{i}^{T}\left(\mathbf{X}^{(l)}\right)^{*}-b_{i}\right] R_{i}^{-1}\left(\mathbf{X}^{(l)}\right) \mathbf{w}_{\tilde{b}_{i}^{*} \mathbf{a}_{i}^{*}}-R_{i}^{-1}\left(\mathbf{X}^{(l)}\right) \\
& \left.\cdot b_{i} \mathbf{a}_{i}^{T}\right\}^{T}
\end{aligned}
$$




$$
\begin{aligned}
& \Longrightarrow \sum_{i=1}^{d_{k}}\left\{R_{i}^{-2}\left(\mathbf{X}^{(l)}\right)\left|\mathbf{a}_{i}^{H} \mathbf{X}^{(l)}-b_{i}\right|^{2} \mathbf{w}_{\widetilde{\mathbf{a}}_{i}}-R_{i}^{-1}\left(\mathbf{X}^{(l)}\right)\right. \\
& \left.\cdot \mathbf{a}_{i} \mathbf{a}_{i}^{H}\right\} \mathbf{X}^{(l+1)}=\sum_{i=1}^{d_{k}}\left\{R_{i}^{-2}\left(\mathbf{X}^{(l)}\right)\left|\mathbf{a}_{i}^{H} \mathbf{X}^{(l)}-b_{i}\right|^{2} \mathbf{w}_{\widetilde{\mathbf{a}}_{i} \tilde{b}_{i}}\right. \\
& \left.-R_{i}^{-1}\left(\mathbf{X}^{(l)}\right) b_{i} \mathbf{a}_{i}\right\}
\end{aligned}
$$

which is equivalent to a standard system of linear equations $F\left(\mathbf{X}^{(l)}\right) \mathbf{X}^{(l+1)}=T\left(\mathbf{X}^{(l)}\right)$ where

$$
\begin{aligned}
& F\left(\mathbf{X}^{(l)}\right) \triangleq \sum_{i=1}^{d_{k}}\left[\mathbf{w}_{\tilde{\mathbf{a}}_{i}} \frac{\left|\mathbf{a}_{i}^{H} \mathbf{X}^{(l)}-b_{i}\right|^{2}}{R_{i}^{2}\left(\mathbf{X}^{(l)}\right)}-\frac{\mathbf{a}_{i} \mathbf{a}_{i}^{H}}{R_{i}\left(\mathbf{X}^{(l)}\right)}\right], \\
& T\left(\mathbf{X}^{(l)}\right) \triangleq \sum_{i=1}^{d_{k}}\left[\mathbf{w}_{\tilde{\mathbf{a}}_{i} \tilde{b}_{i}} \frac{\left|\mathbf{a}_{i}^{H} \mathbf{X}^{(l)}-b_{i}\right|^{2}}{R_{i}^{2}\left(\mathbf{X}^{(l)}\right)}-\frac{b_{i} \mathbf{a}_{i}}{R_{i}\left(\mathbf{X}^{(l)}\right)}\right] .
\end{aligned}
$$

On the basis of (29)-(32), we repeat the iterative process and obtain a series of values $\mathbf{X}^{(l)}$ and $\mathbf{X}^{(l+1)}$ to approximate to accurate value. The iteration continues until $\| \mathbf{X}^{(l+1)}$ $\mathbf{X}^{(l)}\left\|_{F} /\right\| \mathbf{X}^{(l+1)} \|_{F}<\delta_{0}$, and $\delta_{0}$ is a given tolerance.

After the iterative process above, we have the final $\mathbf{X}^{(l)}$, approximate to accurate value. Assume that

$$
\mathbf{X}_{\mathrm{opt}}=\mathbf{X}^{(l)}=\widehat{\mathbf{V}}_{k}^{(l)} \mathbf{s}_{k}, \quad k \in\{1,2, \ldots, K\} .
$$

Finally, the estimated TPC matrix $\widehat{\mathbf{V}}_{k, \text { opt }}=\widehat{\mathbf{V}}_{k}^{(l)}(k \in$ $\{1,2, \ldots, K\})$ can be readily obtained. Hence, the series of estimated values $\widehat{\mathbf{V}}_{k, \text { opt }}(k \in\{1,2, \ldots, K\})$ are optimized to the true value of the TPC matrix $\mathbf{V}_{k}(k \in\{1,2, \ldots, K\})$ by using WTLS-based IA optimization method.

The procedures of the above iteration are outlined in the following algorithm which is presented to solve (24).

\section{Algorithm 1 (WTLS-cased IA optimization algorithm).}

(1) Start with arbitrary TPC matrix $\mathbf{V}_{k}$ and receiving filter matrix $\mathbf{U}_{k}$.

(2) Set the data matrix with CSI error $\mathbf{D} \triangleq\left[\begin{array}{ll}\mathbf{A} & \mathbf{B}\end{array}\right]=$ $\left[\begin{array}{ll}\mathbf{U}_{k}^{H} \widehat{\mathbf{H}}_{k k} & \mathbf{U}_{k}^{H} \widehat{\mathbf{H}}_{k k} \widehat{\mathbf{V}}_{k} \mathbf{s}_{k}\end{array}\right]$, the measurement error $\widetilde{\mathbf{D}}=$ $\left[\mathbf{U}_{k}^{H} \widetilde{\mathbf{H}}_{k k} \widehat{\mathbf{s}}_{k}-\mathbf{U}_{k}^{H} \widehat{\mathbf{H}}_{k k} \widehat{\mathbf{V}}_{k} \mathbf{s}_{k}\right]$, and the extended matrix of the approximate estimation $\widehat{\mathbf{V}}_{k} \mathbf{s}_{k}: \widehat{\mathbf{X}} \triangleq$ $\left[\begin{array}{c}\widehat{\mathbf{V}}_{k} \boldsymbol{s}_{k} \\ -\mathrm{I}\end{array}\right], k \in\{1,2, \ldots, K\}$. Caculate the error weights information $\left\{\mathbf{W}_{i}\right\}_{i=1}^{d_{k}}$. According to (4) and (5), give the variances of CSI error, while a convergence tolerance $\delta_{0}$ of WTLS estimation algorithm should also be given.

(3) According to (13), obtain an initial value $\mathbf{X}^{(1)}$ of WTLS estimation.

(4) Begin iteration, and set the iteration counter $l=1$.

(5) Let $R_{i}\left(\mathbf{X}^{(l)}\right)=\left(\widehat{\mathbf{X}}^{(l)}\right)^{H} \mathbf{W}_{i} \widehat{\mathbf{X}}^{(l)}$, for $i=1,2, \ldots, d_{k}$.

(6) Compute the residual matrix $Q\left(\mathbf{X}^{(l)}\right)=\mathbf{A} \mathbf{X}^{(l)}-\mathbf{B}$.
(7) Define the iterative approximation function as $G\left(\mathbf{X}^{(l+1)}, \mathbf{X}^{(l)}\right)=0$ and work out $\mathbf{X}^{(l+1)}$.

(8) Increase the iteration counter $l=l+1$.

(9) Repeat steps 5 through 8 until $\| \mathbf{X}^{(l+1)} \quad$ $\mathbf{X}^{(l)}\left\|_{F} /\right\| \mathbf{X}^{(l+1)} \|_{F}<\delta_{0}$.

(10) The solution of optimal WTLS estimation is $\mathbf{X}_{\text {opt }}=$ $\mathbf{X}^{(l)}$.

(11) Finally, according to (13) and constraint of TPC matrix [5], obtain the TPC matrix $\mathbf{V}_{k}$. Let $\mathbf{V}_{k}=$ $\widehat{\mathbf{V}}_{k, \text { opt }}, k \in\{1,2, \ldots, K\}$.

(12) Calculate $\mathbf{U}_{k}$ from $\mathbf{U}_{k} \sum_{l \neq k} \mathbf{H}_{k l} \mathbf{V}_{l}=\mathbf{0}$ or MMSE method [5].

In this WTLS-based IA optimization scheme, the sum rate result for stream $s$ of user $k$ is given by (34) where the $\left(\mathbf{H}_{k k}-\widetilde{\mathbf{H}}_{k k}\right) \mathbf{V}_{k, s} \mathbf{V}_{k, s}^{H}\left(\mathbf{H}_{k k}-\widetilde{\mathbf{H}}_{k k}\right)^{H}$ in (34) is the desired signal, and the first, second, third, and fourth terms in (35) represent the interference purely by channel uncertainty, interstream interference, other user interferences, and AWGN, respectively:

$$
\begin{aligned}
& R_{k, \text { sum }}^{(s)}=\log _{2} \operatorname{det}(\mathbf{I} \\
& \left.\quad+\widehat{\mathbf{Z}}_{k, s}^{-1}\left(\mathbf{H}_{k k}-\widetilde{\mathbf{H}}_{k k}\right) \mathbf{V}_{k, s} \mathbf{V}_{k, s}^{H}\left(\mathbf{H}_{k k}-\widetilde{\mathbf{H}}_{k k}\right)^{H}\right),
\end{aligned}
$$

where

$$
\begin{aligned}
& \widehat{\mathbf{Z}}_{k, s}=\widetilde{\mathbf{H}}_{k k} \mathbf{V}_{k, s} \mathbf{V}_{k, s}^{H} \widetilde{\mathbf{H}}_{k k}^{H}+\sum_{\substack{t=1 \\
t \neq s}}^{d_{k}} \mathbf{H}_{k k} \mathbf{V}_{k, t} \mathbf{V}_{k, t}^{H} \mathbf{H}_{k k}^{H} \\
& +\sum_{\substack{l=1 \\
l \neq k}}^{K} \sum_{t=1}^{d_{l}} \mathbf{H}_{k l} \mathbf{V}_{l, t} \mathbf{V}_{l, t}^{H} \mathbf{H}_{k l}^{H}+P_{k, \text { noise }} \mathbf{I} .
\end{aligned}
$$

$\mathbf{V}_{k, s}$ is the TPC for stream $s$ of user $k$ and $P_{k \text {,noise }}$ is the power of noise at user $k$. The numerator of (34) is the desired signal power, while the denominator $\widehat{\mathbf{Z}}_{k, s}$ of (34) is interferences plus noise [11]. And the first, second, third, and fourth terms in $\widehat{\mathbf{Z}}_{k, s}$ of the right-hand side of (35) represent the internal perturbation purely by CSI error, interstream interference, other user interferences, and AWGN, respectively. Hence, all sum rate of $K$-user IA scheme can be derived easily as follows:

$$
R_{\text {sum }}=\sum_{k=1}^{K} \sum_{s=1}^{d_{k}} R_{k, \text { sum }}^{(s)} .
$$

3.3. Convergence Issue. In this part, we present the convergence issue of the above iterative algorithm for the WTLS optimization in the previous part. The convergence issue equals WTLS estimation $\mathbf{X}$ which converges to $\mathbf{X}_{0}$ or $\widehat{\mathbf{X}}$ which converges to $\mathbf{X}_{\text {tru }}$ in probability $[23,29,30]$.

Define the variance of each element of measurement error matrix $\widetilde{\mathbf{D}}$

$$
\sigma_{i, j}^{2} \triangleq E\left\{\widetilde{d}_{i, j}^{2}\right\}, \quad i=1,2, \ldots, d_{k}, j=1,2, \ldots, N_{t}+1 .
$$


We allow some of $\sigma_{i, j}$ to be equal to 0 . The following assumptions (I) to (VI) are satisfied:

(I) For a fixed $J \subset\left\{1,2, \ldots, N_{t}+1\right\}$, every $j \notin J$ and every $i=1,2, \ldots, d_{k}$ satisfy $\sigma_{i, j}=0$.

(II) There exists $\varepsilon>0$ such that, for every $i=1,2, \ldots, d_{k}$, $\lambda_{\min }\left(\mathbf{W}_{i}\right) \geq \varepsilon^{2}$.

(III) The elements of $\widehat{\mathbf{X}}$ are nonzero vector.

(IV) There exists $\alpha \geq 2$ with $\alpha \geq|J|-1$ such that $\sup _{(i \geq 1, j \in J)} E\left\{\left|\widetilde{d}_{i, j}\right|^{2 \alpha}\right\}<\infty$.

(V) $\sum_{d_{k}=d_{0}}^{N}\left[\lambda_{\text {max }}\left(\mathbf{A}^{H} \mathbf{A}\right) / \lambda_{\text {min }}^{2}\left(\mathbf{A}^{H} \mathbf{A}\right)\right]^{r}<\infty, d_{0} \geq 1$, and $N$ is infinite.

(VI) $\sum_{d_{k}=d_{0}}^{N}\left[\sqrt{d_{k}} / \lambda_{\min }\left(\mathbf{A}^{H} \mathbf{A}\right)\right]^{r}<\infty, d_{0} \geq 1$, and $N$ is infinite.

Then the WTLS optimization estimation $\mathbf{X}$ converges to $\mathbf{X}_{0}$ almost surely (a.s.), as $d_{k}$ is large enough. The details of the proofs of convergence and consistency can be found in [23]. And the convergence, availability, and consistency of WTLS optimization estimation are well investigated [18, 23, 29, 30].

From above statements, we find that if $d_{k}$ is large enough, estimation $\mathbf{X}$ converges to $\mathbf{X}_{0}$ with probability tending to 1. That means the number of rows of the data matrix $\mathbf{D}$ must be so large that estimation $\mathbf{X}$ converges to $\mathbf{X}_{0}$ with $100 \%$ probability. Mathematically, it seems feasible. However, realistically, it is unfeasible in interference management of multiuser MIMO communication system. Since the DoF cannot be infinite. A compromise is reached by the extending the row spacef of the data matrix $\mathbf{D}$

$$
\mathbf{D} \triangleq\left[\begin{array}{cc}
\mathbf{U}_{k 1}^{H} \widehat{\mathbf{H}}_{k k} & \mathbf{U}_{k 1}^{H} \widehat{\mathbf{H}}_{k k} \mathbf{V}_{k 1} \mathbf{s}_{k} \\
\mathbf{U}_{k 2}^{H} \widehat{\mathbf{H}}_{k k} & \mathbf{U}_{k 2}^{H} \widehat{\mathbf{H}}_{k k} \mathbf{V}_{k 2} \mathbf{s}_{k} \\
\vdots & \vdots \\
\mathbf{U}_{k n}^{H} \widehat{\mathbf{H}}_{k k} & \mathbf{U}_{k n}^{H} \widehat{\mathbf{H}}_{k k} \mathbf{V}_{k n} \mathbf{s}_{k} \\
\vdots & \vdots
\end{array}\right]
$$

where $\mathbf{D} \in \mathbb{C}^{N \times\left(N_{t}+1\right)}$ and $\mathbf{V}_{k n}$ and $\mathbf{U}_{k n}(n=1,2, \ldots)$ are the arbitrary initialized TPC matrix and receiving filter matrix, respectively. The relevant evaluation of $n$ will be discussed in the next section.

\section{Numerical Results}

In this section, simulations are conducted to evaluate the performance of our WTLS-based IA scheme presented in Section 3 for multiuser MIMO interference system with imperfect CSI.

Without loss of generality, the CSI errors are assumed to be i.i.d. zero-mean complex Gaussian, and Gaussian input is considered. size of data matrix $\mathbf{D}$ is fixed in Figures 2 to 5; that is, the value of $n$ in (38) is fixed (here we set $n=64$ ), while the WTLS estimation convergence tolerance $\delta_{0}=10^{-5}$. The parameters $\alpha$ and $\beta$ indicate the variance of CSI errors for direct link and interference links, respectively. For given

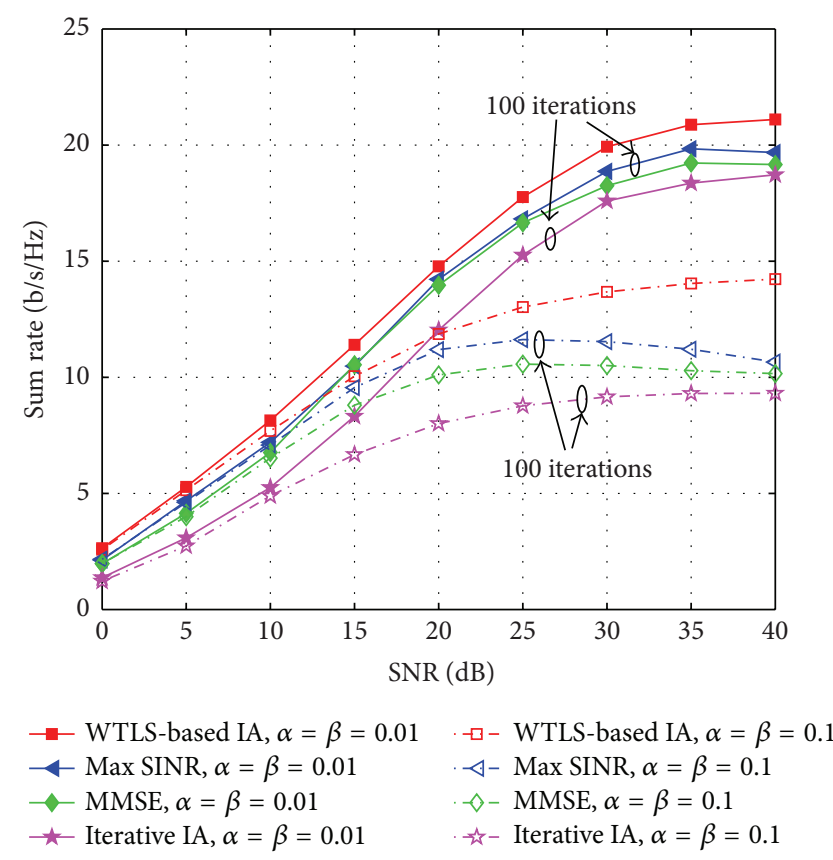

FIGURE 2: The sum rate performance of different IA schemes with imperfect CSI for $K=3$ and $N_{t}=N_{r}=3$ and for the cases $\alpha=\beta=$ 0.1 and $\alpha=\beta=0.01$.

$\alpha$ and $\beta$, we first generate $\widetilde{\mathbf{H}}_{k k}$ and $\widetilde{\mathbf{H}}_{k l}$ randomly according to a zero-mean complex Gaussian distribution from (4) and (5). After $\widetilde{\mathbf{H}}_{k k}$ and $\widetilde{\mathbf{H}}_{k l}$ are generated as such, we generate $\widehat{\mathbf{H}}_{k k}$ and $\widehat{\mathbf{H}}_{k l}$ according to (3) to (6), and the true channel is determined by (3). In each plot presented in this section, the sum rate performance is computed via Monte Carlo simulations using 1000 independent channel realizations.

Figure 2 shows the sum rate performance of different IA schemes with imperfect CSI for the cases $\alpha=\beta=0.1$ and $\alpha=\beta=0.01$, respectively, where we have $K=3$ users and $N_{t}=N_{r}=3$ antennas at each node. The theoretical maximum DoF which is 4 can be achieved, which means one of the transmitters can obtain $2 \mathrm{DoF}$ and the other two are with $1 \mathrm{DoF}$. That is to say, this system is unsymmetrical MIMO interference network. It can be observed that the sum rates of all schemes increase as the variance of CSI error decreases. However, the sum rates of all schemes have the error-floor effect because of the internal perturbation purely by CSI error and the interstream interference. From Figure 2, it is seen that the proposed WTLS-based IA scheme outperforms the Max SINR [5], MMSE [13, 14], and Iterative IA [5] (i.e., CJ08 algorithm) schemes for all SNR under different variances of CSI error scenarios. The Max SINR and MMSE schemes show good performance almost comparable to our proposed WTLS-based IA scheme at low SNR. However, as the SNR increases, the sum rates of Max SINR and MMSE schemes increase at slower rate than that of the WTLS-based IA scheme. Particularly, the sum rate of Max SINR scheme degrades to that of MMSE scheme as the SNR increases (these two schemes themselves converge as SNR increases), while both of them still outperform Iterative IA scheme. 


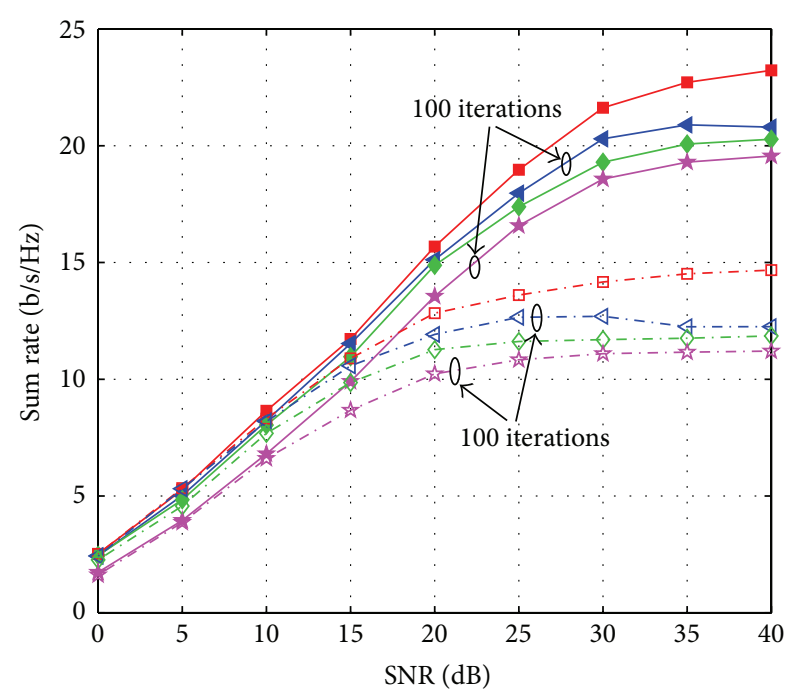

$\begin{array}{ll}\rightarrow \text { WTLS-based IA, } \alpha=\beta=0.01 & - \text { - WTLS-based IA, } \alpha=\beta=0.1 \\ \varangle \text { Max SINR, } \alpha=\beta=0.01 & \triangleleft-\text { Max SINR, } \alpha=\beta=0.1 \\ \neg \text { MMSE, } \alpha=\beta=0.01 & \checkmark-\text { MMSE, } \alpha=\beta=0.1 \\ \star \text { Iterative IA, } \alpha=\beta=0.01 & - \text { - Iterative IA, } \alpha=\beta=0.1\end{array}$

FIGURE 3: The sum rate performance of different IA schemes with imperfect CSI for $K=3$ and $N_{t}=N_{r}=4$ and for the cases $\alpha=\beta=$ 0.1 and $\alpha=\beta=0.01$.

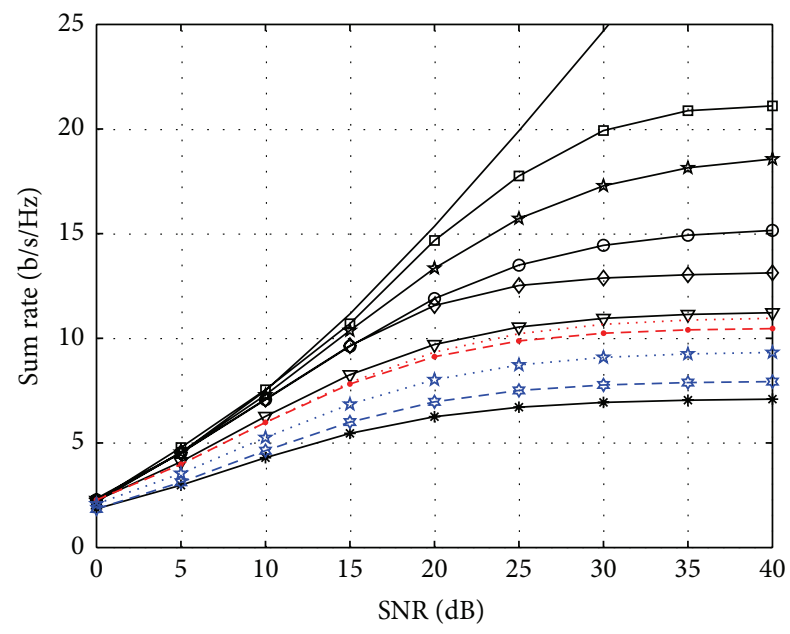

$$
\begin{aligned}
& \text { - Perfect CSI } \\
& \square \text { WTLS-based IA, } \alpha=\beta=0.01 \\
& \text { \# WTLS-based IA, } \alpha=\beta=0.02 \\
& - \text { WTLS-based IA, } \alpha=\beta=0.05 \\
& \checkmark \text { WTLS-based IA, } \alpha=\beta=0.1 \\
& \nabla \text { WTLS-based IA, } \alpha=\beta=0.2 \\
& * \text { WTLS-based IA, } \alpha=\beta=0.5 \\
& \text { WTLS-based IA, } \alpha=0.2, \beta=0.3 \\
& \text { - - WTLS-based IA, } \alpha=0.2, \beta=0.5 \\
& \text {. W. WTLS-based IA, } \alpha=0.3, \beta=0.2 \\
& \text { - WTLS-based IA, } \alpha=0.5, \beta=0.2
\end{aligned}
$$

FIgURE 4: The sum rate of the proposed WTLS-based IA scheme for $K=3$ users and $N_{t}=N_{r}=3$ with different CSI uncertainties.

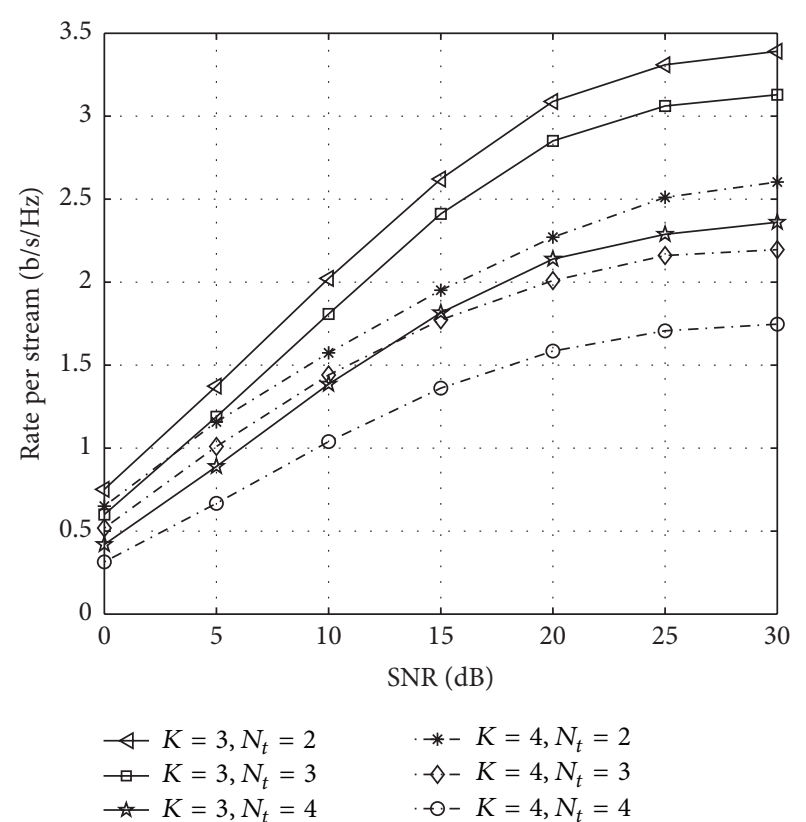

FIGURE 5: The achievable per-stream rate of the proposed WTLSbased IA scheme for the case $\alpha=\beta=0.1$.

Generally, Figure 2 shows that there is a considerable gain by using our WTLS-based IA scheme especially under a serious CSI error scenario. Meanwhile, the number of iterations in WTLS-based IA scheme is not more than 10 [19]. It is much less than 100 iterations in both Max SINR and Iterative IA schemes and still less than 16 iterations $[13,14]$ in MMSE scheme (this MMSE scheme is slightly more complicated as Lagrange multipliers need to be computed to design the transmit beamformers [31]).

Figure 3 portrays the sum rate performance of different IA schemes with imperfect CSI for the cases $\alpha=\beta=0.1$ and $\alpha=\beta=0.01$, respectively, where we have $K=3$ users and $N_{t}=N_{r}=4$ antennas at each node. The theoretical maximum DoF is 6 which can be achieved, which means each transmitter is with $2 \mathrm{DoF}$. That is to say, this system is symmetrical MIMO interference network. Comparing to Figure 2, although the sum rates of all schemes increase as the variance of CSI error decreases, the increasement of the sum rates of all schemes is limited due to the more inter-stream interference with imperfect CSI. It can be observed that the proposed WTLS-based IA scheme still outperforms the other schemes for all SNR under different variances of CSI error scenarios. Our proposed WTLS-based IA scheme can achieve a better performance than the other schemes even at low SNR. Generally, the proposed WTLS-based IA scheme is proven to have good performance under the scenario of symmetrical multiple interstream interference.

Figure 4 illustrates the achievable sum rate of the proposed WTLS-based IA scheme for different variances of CSI error, where we have $K=3$ users and $N_{t}=N_{r}=3$ antennas at each node. It is seen that the achievable sum rate decreases as the variance of CSI error increases. When the variance of 


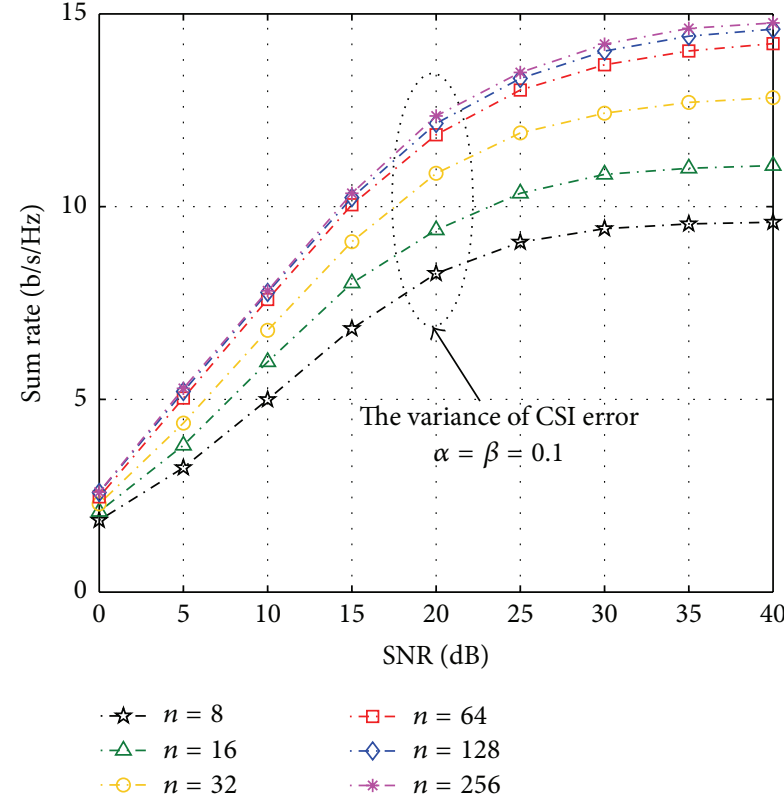

(a) Sum rate versus SNR for $\alpha=\beta=0.1$

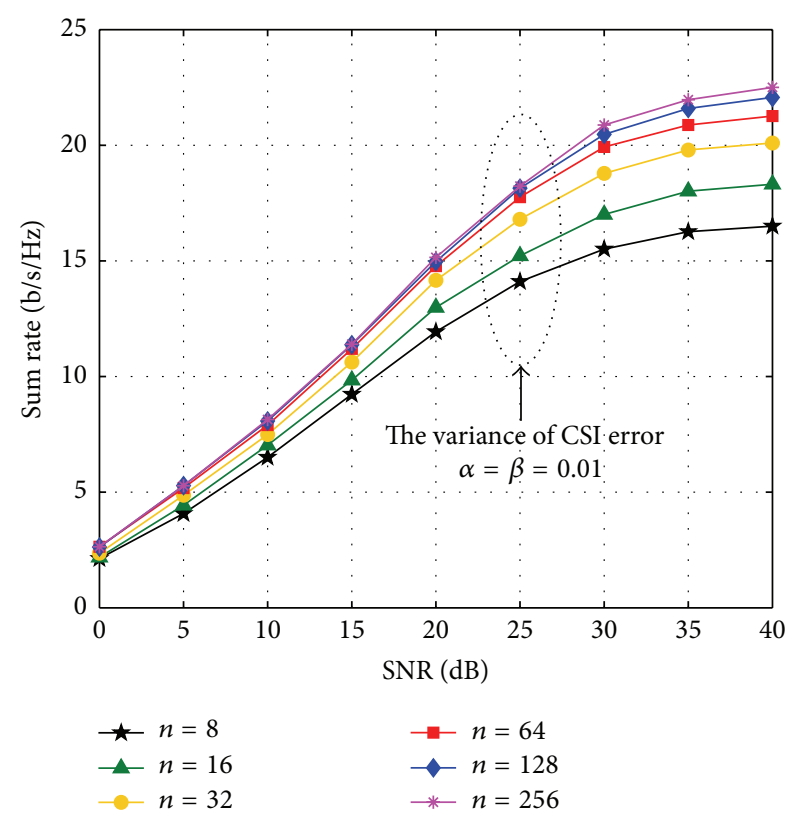

(b) Sum rate versus SNR for $\alpha=\beta=0.01$

FiguRE 6: The sum rate performance of the proposed WTLS-based IA scheme with different sizes of data matrix $\mathbf{D}$ for $K=3, N_{t}=N_{r}=3$.

CSI error becomes sufficiently small, the achievable sum rate surely approaches the one with perfect CSI. The starlike and dotted lines represent the achievable sum rate of the proposed WTLS-based IA scheme with different variances of CSI error for direct link $\alpha$ and interference links $\beta$, respectively. It can be observed that the sum rate decays as the variance of CSI error only for direct link or interference links increases. The increasement of the variance of CSI error for direct link $\alpha$ has a much worse effect on the achievable sum rate than that of the variance of CSI error for interference links $\beta$. This leads to a conclusion that transmitter can achieve a desired transmission rate by allocating minimum required resources according to CSI.

Figure 5 describes achievable per-stream rate of the proposed WTLS-based IA scheme versus SNR in $K$-user interference channels, where we have $\alpha=\beta=0.1$. As shown in Figure 5, the achievable per-stream rate monotonically increases, and it saturates in the high SNR regime. However, because of the negative effect of interuser interference, the achievable per-stream rate monotonically decreases as the number of the users increases. It can be also observed that the per-stream rate performance of single stream case for each user is much better than the case of the multiple streams case for each user. The reason is that interstream interference has a negative effect on the rate performance under the CSI error scenario. Generally, under a CSI error scenario (especially a serious CSI error scenario), the more users or antennas the communication system has, the worse the rate performance will be.

Figures 6(a) and 6(b) show the sum rate performance of the proposed WTLS-based IA scheme with different sizes of data matrix $\mathbf{D}$ for $\alpha=\beta=0.1$ and $\alpha=\beta=0.01$, respectively, where we have $K=3$ users and $N_{t}=N_{r}=3$ antennas at each node. It can be observed that the sum rate increases as the size of data matrix $\mathbf{D}$ magnifies. In other words, when the value of $n$ in (38) increases, the sum rate performance of the proposed WTLS-based IA scheme increases. From both Figures 6(a) and 6(b), it can be found that the sum rate performance increases more slowly when the value of $n$ is larger than 64 . The sum rate begins to converge as $n=128$. These observations confirm that if the number of rows of the data matrix $\mathbf{D}$ is large enough, estimation $\mathbf{X}$ converges to $\mathbf{X}_{0}$ with probability tending to 1 .

\section{Conclusions}

In this paper, we have considered the sum rate performance of different IA schemes under the condition of CSI uncertainties. We have established an EIV-based system model of IA with imperfect CSI and presented an IA scheme based on WTLS error analysis algorithm for turning the imperfect CSI-based IA optimization (i.e., constrained optimization problem) into an equivalently unconstrained optimization problem in the transmitted signal estimation. Meanwhile, the convergence of the proposed WTLS-based IA scheme is presented as well. Numerical results have shown that the proposed WTLS-based IA scheme can effectively improve the sum rate performance of the MIMO interference channel system compared with several existing IA schemes under different scenarios of CSI error. Numerical results have also shown that the larger the number of rows of the data matrix $\mathbf{D}$ is, the higher the accuracy of estimation $\mathbf{X}$ is. 


\section{Appendix}

\section{Conjugate Gradient of $f(\mathbf{X})$}

$q_{i}^{H}(\mathbf{X}), q_{i}(\mathbf{X})$, and $R_{i}(\mathbf{X})$ are all the functions of argument $\mathbf{X}$. For the sake of convenience, they can be expressed by $\mathbf{X}$ as follows:

$$
\begin{aligned}
& q_{i}^{H}(\mathbf{X})=(\mathbf{D} \widehat{\mathbf{X}})_{i,:}=\left(\left[\begin{array}{ll}
\mathbf{A} & \mathbf{B}
\end{array}\right]\left[\begin{array}{c}
\mathbf{X} \\
-\mathbf{I}
\end{array}\right]\right)_{i,:}=(\mathbf{A X}-\mathbf{B})_{i,:} \\
& =\left(\mathbf{A}_{i,:} \mathbf{X}-\mathbf{B}_{i,:}\right)=\mathbf{a}_{i}^{H} \mathbf{X}-\mathbf{b}_{i}^{H}, \\
& q_{i}(\mathbf{X})=\left(\mathbf{a}_{i}^{H} \mathbf{X}-\mathbf{b}_{i}^{H}\right)^{H}=\mathbf{X}^{H} \mathbf{a}_{i}-\mathbf{b}_{i}, \\
& R_{i}(\mathbf{X})=\widehat{\mathbf{X}}^{H} \mathbf{W}_{i} \widehat{\mathbf{X}}=\left[\begin{array}{ll}
\mathbf{X}^{H} & -\mathbf{I}
\end{array}\right] \operatorname{cov}\left(\tilde{\mathbf{d}}_{i}\right)\left[\begin{array}{c}
\mathbf{X} \\
-\mathbf{I}
\end{array}\right] \\
& =\left[\begin{array}{ll}
\mathbf{X}^{H} & -\mathbf{I}
\end{array}\right]\left[\begin{array}{cc}
\operatorname{cov}\left(\widetilde{\mathbf{a}}_{i}\right) & \operatorname{cov}\left(\widetilde{\mathbf{a}}_{i}, \widetilde{\mathbf{b}}_{i}\right) \\
\operatorname{cov}\left(\widetilde{\mathbf{b}}_{i}, \widetilde{\mathbf{a}}_{i}\right) & \operatorname{cov}\left(\widetilde{\mathbf{b}}_{i}\right)
\end{array}\right]\left[\begin{array}{c}
\mathbf{X} \\
-\mathbf{I}
\end{array}\right] .
\end{aligned}
$$

Obviously, $f(\mathbf{X})$ is a scalar function. We can treat $f(\mathbf{X})$ as function with respect to $q_{i}(\mathbf{X})$ and $R_{i}(\mathbf{X})$. According to the conjugate gradient method for unconstraint optimization and the chain rule of matrix derivative $[27,28]$, we have

$$
\begin{aligned}
& \frac{\partial f(\mathbf{X})}{\partial \mathbf{X}^{*}}=\sum_{i=1}^{d_{k}} \frac{\partial f\left(q_{i}, R_{i}\right)}{\partial \mathbf{X}^{*}}=\sum_{i=1}^{d_{k}}\left[\frac{\partial q_{i}^{T}(\mathbf{X})}{\partial \mathbf{X}^{*}} \frac{\partial\left(q_{i}^{H} R_{i}^{-1} q_{i}\right)}{\partial q_{i}}\right. \\
& \left.+\frac{\partial R_{i}^{T}(\mathbf{X})}{\partial \mathbf{X}^{*}} \frac{\partial\left(q_{i}^{H} R_{i}^{-1} q_{i}\right)}{\partial R_{i}}\right],
\end{aligned}
$$

where $q_{i}(\mathbf{X})$ is a vector, while $R_{i}(\mathbf{X})$ is matrix. Note that, in the EIV-based IA system, $\mathbf{s}_{k}$ and $\widehat{\mathbf{X}}$ are vectors; thus the solution of $\mathbf{X}$ is univariate case. In this case, $R_{i}(\mathbf{X})$ is a single component matrix, while $q_{i}(\mathbf{X}), \mathbf{b}_{i}$, and $\widetilde{\mathbf{b}}_{i}$ are all single component vectors. According to the derivative rules of function with vector argument $[27,28]$, the $\partial\left(q_{i}^{H} R_{i}^{-1} q_{i}\right) / \partial q_{i}$ in the first term of (A.4) can be rewritten as

$$
\frac{\partial\left(q_{i}^{H} R_{i}^{-1} q_{i}\right)}{\partial q_{i}}=\left[R_{i}^{-1}(\mathbf{X})\right]^{T} q_{i}^{*}(\mathbf{X})=R_{i}^{-1}(\mathbf{X}) q_{i}^{*}(\mathbf{X})
$$

According to the derivative rules of function with matrix argument relevant to matrix inverse [28], $\partial\left(q_{i}^{H} R_{i}^{-1} q_{i}\right) / \partial q_{i}^{H}$ in the second term of (A.4) can be written as

$$
\begin{aligned}
& \frac{\partial\left(q_{i}^{H} R_{i}^{-1} q_{i}\right)}{\partial R_{i}} \\
& \quad=-\left[R_{i}^{-1}(\mathbf{X})\right]^{T} q_{i}^{*}(\mathbf{X}) q_{i}^{T}(\mathbf{X})\left[R_{i}^{-1}(\mathbf{X})\right]^{T} \\
& \quad=-R_{i}^{-1}(\mathbf{X}) q_{i}^{*}(\mathbf{X}) q_{i}(\mathbf{X}) R_{i}^{-1}(\mathbf{X})
\end{aligned}
$$

Then (A.4) can be rewritten as follows:

$$
\begin{gathered}
\frac{\partial f(\mathbf{X})}{\partial \mathbf{X}^{*}}=\sum_{i=1}^{d_{k}}\left\{\frac{\partial q_{i}^{T}(\mathbf{X})}{\partial \mathbf{X}^{*}} R_{i}^{-1}(\mathbf{X}) q_{i}^{*}(\mathbf{X})-\frac{\partial R_{i}^{T}(\mathbf{X})}{\partial \mathbf{X}^{*}}\right. \\
\left.\cdot R_{i}^{-1}(\mathbf{X}) q_{i}^{*}(\mathbf{X}) q_{i}^{T}(\mathbf{X}) R_{i}^{-1}(\mathbf{X})\right\}=\sum_{i=1}^{d_{k}}\left\{R_{i}^{-1}(\mathbf{X})\right. \\
\left.\cdot q_{i}^{*}(\mathbf{X})\left[\frac{\partial q_{i}^{T}(\mathbf{X})}{\partial \mathbf{X}^{*}}+q_{i}(\mathbf{X}) R_{i}^{-1}(\mathbf{X}) \frac{\partial R_{i}^{T}(\mathbf{X})}{\partial \mathbf{X}^{*}}\right]\right\} .
\end{gathered}
$$

We can derive (A.7) by deducing the terms of $\partial q_{i}(\mathbf{X}) / d \mathbf{X}^{*}$ and $\partial R_{i}(\mathbf{X}) / \partial \mathbf{X}^{*}$. Firstly, the derivative term of $\partial q_{i}(\mathbf{X}) / \partial \mathbf{X}^{*}$ can be derived from (A.2)

$$
\frac{\partial q_{i}^{T}(\mathbf{X})}{\partial \mathbf{X}^{*}}=\left[\frac{\partial q_{i}(\mathbf{X})}{\partial \mathbf{X}^{*}}\right]^{T}=\left[\frac{d\left(\mathbf{X}^{H} \mathbf{a}_{i}-b_{i}\right)}{d \mathbf{X}^{*}}\right]^{T}=\mathbf{a}_{i}^{T} .
$$

Secondly, derivative term of $\partial R_{i}(\mathbf{X}) / \partial \mathbf{X}$ can be derived from (A.3)

$$
\begin{aligned}
& \frac{\partial R_{i}^{T}(\mathbf{X})}{\partial \mathbf{X}^{*}}=\left[\frac{\partial\left(\left[\mathbf{X}^{H}-\mathbf{I}\right] \mathbf{W}_{i}\left[\begin{array}{c}
\mathbf{X} \\
-\mathbf{I}
\end{array}\right]\right)}{\partial \mathbf{X}^{*}}\right]^{T} \\
& \quad=\left\{\frac{\partial\left(\left[\mathbf{X}^{H}-\mathbf{I}\right]\right)}{\partial \mathbf{X}^{*}} \mathbf{W}_{i}\left[\begin{array}{c}
\mathbf{X} \\
-\mathbf{I}
\end{array}\right]\right\}^{T} \\
& =\left\{\left[\begin{array}{ll}
\mathbf{I} & \mathbf{0}
\end{array}\right]\left[\begin{array}{cc}
\operatorname{cov}\left(\widetilde{\mathbf{a}}_{i}\right) & \operatorname{cov}\left(\widetilde{\mathbf{a}}_{i}, \widetilde{b}_{i}\right) \\
\operatorname{cov}\left(\widetilde{b}_{i}, \widetilde{\mathbf{a}}_{i}\right) & \operatorname{cov}\left(\widetilde{b}_{i}\right)
\end{array}\right]\left[\begin{array}{c}
\mathbf{X} \\
-\mathbf{I}
\end{array}\right]\right\}^{T} \\
& =\left[\operatorname{cov}\left(\widetilde{\mathbf{a}}_{i}\right) \mathbf{X}-\operatorname{cov}\left(\widetilde{\mathbf{a}}_{i}, \widetilde{b}_{i}\right)\right]^{T} \\
& =\mathbf{X}^{T} \operatorname{cov}\left(\widetilde{\mathbf{a}}_{i}^{*}\right)-\operatorname{cov}\left(\widetilde{b}_{i}^{*}, \widetilde{\mathbf{a}}_{i}^{*}\right) .
\end{aligned}
$$

Finally, substituting (A.8) and (A.9) into (A.7), we obtain the conjugate gradient of $f(\mathbf{X})$

$$
\begin{gathered}
\frac{\partial f(\mathbf{X})}{\partial \mathbf{X}^{*}}=\sum_{i=1}^{d_{k}}\left\{R _ { i } ^ { - 1 } ( \mathbf { X } ) q _ { i } ^ { * } ( \mathbf { X } ) \left[\mathbf{a}_{i}^{T}\right.\right. \\
-q_{i}(\mathbf{X}) R_{i}^{-1}(\mathbf{X}) \mathbf{X}^{T} \operatorname{cov}\left(\widetilde{\mathbf{a}}_{i}^{*}\right) \\
\left.\left.+q_{i}(\mathbf{X}) R_{i}^{-1}(\mathbf{X}) \operatorname{cov}\left(\widetilde{b}_{i}^{*}, \widetilde{\mathbf{a}}_{i}^{*}\right)\right]\right\} .
\end{gathered}
$$

\section{Disclosure}

Zhengmin Kong and Shixin Peng should be considered cofirst authors.

\section{Conflict of Interests}

The authors declare that there is no conflict of interests regarding the publication of this paper. 


\section{Authors' Contribution}

Zhengmin Kong and Shixin Peng contributed equally to this work.

\section{Acknowledgment}

This work was supported in part by the National Natural Science Foundations of China under Grant 61201168.

\section{References}

[1] S. A. Jafar and S. Shamai, "Degrees of freedom region of the $\mathrm{MIMO}_{X}$ channel," IEEE Transactions on Information Theory, vol. 54, no. 1, pp. 151-170, 2008.

[2] V. R. Cadambe and S. A. Jafar, "Interference alignment and degrees of freedom of the $K$-user interference channel," IEEE Transactions on Information Theory, vol. 54, no. 8, pp. 34253441, 2008.

[3] T. Gou and S. A. Jafar, "Degrees of freedom of the $K$ user $M \times N$ MIMO interference channel," IEEE Transactions on Information Theory, vol. 56, no. 12, pp. 6040-6057, 2010.

[4] H. Yu and Y. Sung, "Least squares approach to joint beam design for interference alignment in multiuser multi-input multioutput interference channels," IEEE Transactions on Signal Processing, vol. 58, no. 9, pp. 4960-4966, 2010.

[5] K. Gomadam, V. R. Cadambe, and S. A. Jafar, "A distributed numerical approach to interference alignment and applications to wireless interference networks," IEEE Transactions on Information Theory, vol. 57, no. 6, pp. 3309-3322, 2011.

[6] L. Zhang, L. Song, M. Ma, Z. Zhang, M. Lei, and B. Jiao, "Interference alignment with differential feedback," IEEE Transactions on Vehicular Technology, vol. 61, no. 6, pp. 2878-2883, 2012.

[7] D. A. Schmidt, C. Shi, R. A. Berry, M. L. Honig, and W. Utschick, "Comparison of distributed beamforming algorithms for MIMO interference networks," IEEE Transactions on Signal Processing, vol. 61, no. 13, pp. 3476-3489, 2013.

[8] Z. Kong, S. Yang, F. Wu, L. Zhong, S. Peng, and L. Hanzo, "An iteratively distributed total-utility mse approach for secure communications in mimo interference channel," IEEE Transactions on Vehicular Technology. Submitted.

[9] S. W. Peters and R. W. Heath Jr., "Cooperative algorithms for MIMO interference channels," IEEE Transactions on Vehicular Technology, vol. 60, no. 1, pp. 206-218, 2011.

[10] H. Farhadi, M. N. Khormuji, C. Wang, and M. Skoglund, "Ergodic interference alignment with noisy channel state information," in Proceedings of the IEEE International Symposium on Information Theory (ISIT '13), pp. 584-588, July 2013.

[11] J. Park, Y. Sung, D. Kim, and H. V. Poor, "Outage probability and outage-based robust beamforming for MIMO interference channels with imperfect channel state information," IEEE Transactions on Wireless Communications, vol. 11, no. 10, pp. 3561-3573, 2012.

[12] H. Shen, B. Li, Y. Luo, and F. Liu, "A robust interference alignment scheme for the MIMO X channel," in Proceedings of the 15th Asia-Pacific Conference on Communications (APCC '09), pp. 241-244, October 2009.

[13] H. Shen, B. Li, M. Tao, and Y. Luo, "The new interference alignment scheme for the MIMO interference channel," in Proceedings of the IEEE Wireless Communications and Networking Conference (WCNC '10), pp. 1-6, April 2010.
[14] H. Shen, B. Li, M. Tao, and X. Wang, "MSE-based transceiver designs for the MIMO interference channel," IEEE Transactions on Wireless Communications, vol. 9, no. 11, pp. 3480-3489, 2010.

[15] H. Huang, V. K. N. Lau, Y. Du, and S. Liu, "Robust lattice alignment for $K$-user MIMO interference channels with imperfect channel knowledge," IEEE Transactions on Signal Processing, vol. 59, no. 7, pp. 3315-3325, 2011.

[16] B. Xie, Y. Li, H. Minn, and A. Nosratinia, "Adaptive interference alignment with CSI uncertainty," IEEE Transactions on Communications, vol. 61, no. 2, pp. 792-801, 2013.

[17] S. M. Razavi and T. Ratnarajah, "Performance analysis of interference alignment under CSI mismatch," IEEE Transactions on Vehicular Technology, vol. 63, no. 9, pp. 4740-4748, 2014.

[18] I. Markovsky, M. L. Rastello, A. Premoli, A. Kukush, and S. Van Huffel, "The element-wise weighted total least-squares problem," Computational Statistics \& Data Analysis, vol. 50, no. 1, pp. 181-209, 2006.

[19] B. Schaffrin and A. Wieser, "On weighted total least-squares adjustment for linear regression," Journal of Geodesy, vol. 82, no. 7, pp. 415-421, 2008.

[20] I. Hnětynková, M. Plešinger, D. M. Sima, Z. Strakoš, and S. van Huffel, "The total least squares problem in $A X \approx B$ : a new classification with the relationship to the classical works," SIAM Journal on Matrix Analysis and Applications, vol. 32, no. 3, pp. 748-770, 2011.

[21] X. Fang, "Weighted total least-squares with constraints: a universal formula for geodetic symmetrical transformations," Journal of Geodesy, vol. 89, no. 5, pp. 459-469, 2015.

[22] G. Pan, Y. Zhou, H. Sun, and W. Guo, "Linear observation based total least squares," Survey Review, vol. 47, no. 340, pp. 18-27, 2015.

[23] A. Kukush and S. V. Huffel, "Consistency of elementwiseweighted total least squares estimator in a multivariate errorsin-variables model AX=B," Metrika, vol. 59, no. 1, pp. 75-97, 2004.

[24] A. Amiri-Simkooei and S. Jazaeri, "Weighted total least squares formulated by standard least squares theory," Journal of Geodetic Science, vol. 2, pp. 113-124, 2012.

[25] V. Mahboub and M. A. Sharifi, "On weighted total least-squares with linear and quadratic constraints," Journal of Geodesy, vol. 87, no. 3, pp. 279-286, 2013.

[26] P. Sprent, "A generalized least-squares approach to linear functional relationships," Journal of the Royal Statistical Society Series B: Methodological, vol. 28, no. 2, pp. 278-297, 1966.

[27] X. Zhang, Matrix Analysis and Applications, Tsinghua University Press, Beijing, China, 2004.

[28] H. Lütkepohl, Handbook of Matrices, John Wiley \& Sons, Chichester, UK, 1996.

[29] A. Kukush, I. Markovsky, and S. V. Huffel, "About the convergence of the computational algorithm for the EW-TLS estimator," Tech. Rep., Department of Eletrial Engineering, Katholieke Universiteit Leuven, Leuven, Belgium, 2002.

[30] S. V. Shklyar, "Conditions for the consistency of the total least squares estimator in an errors-in-variables linear regression model," Theory of Probability and Mathematical Statistics, vol. 83, pp. 175-190, 2011.

[31] C. Wilson and V. Veeravalli, "A convergent version of the Max SINR algorithm for the MIMO interference channel," IEEE Transactions on Wireless Communications, vol. 12, no. 6, pp. 2952-2961, 2013. 


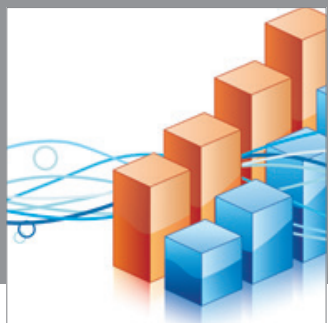

Advances in

Operations Research

mansans

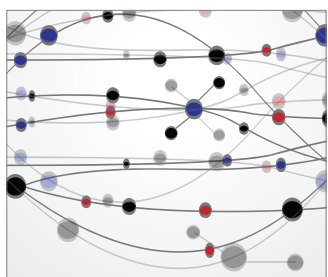

The Scientific World Journal
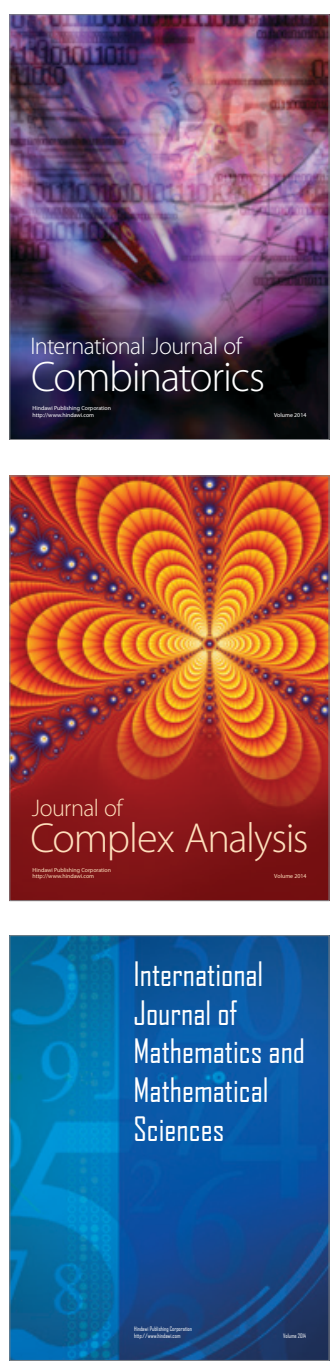
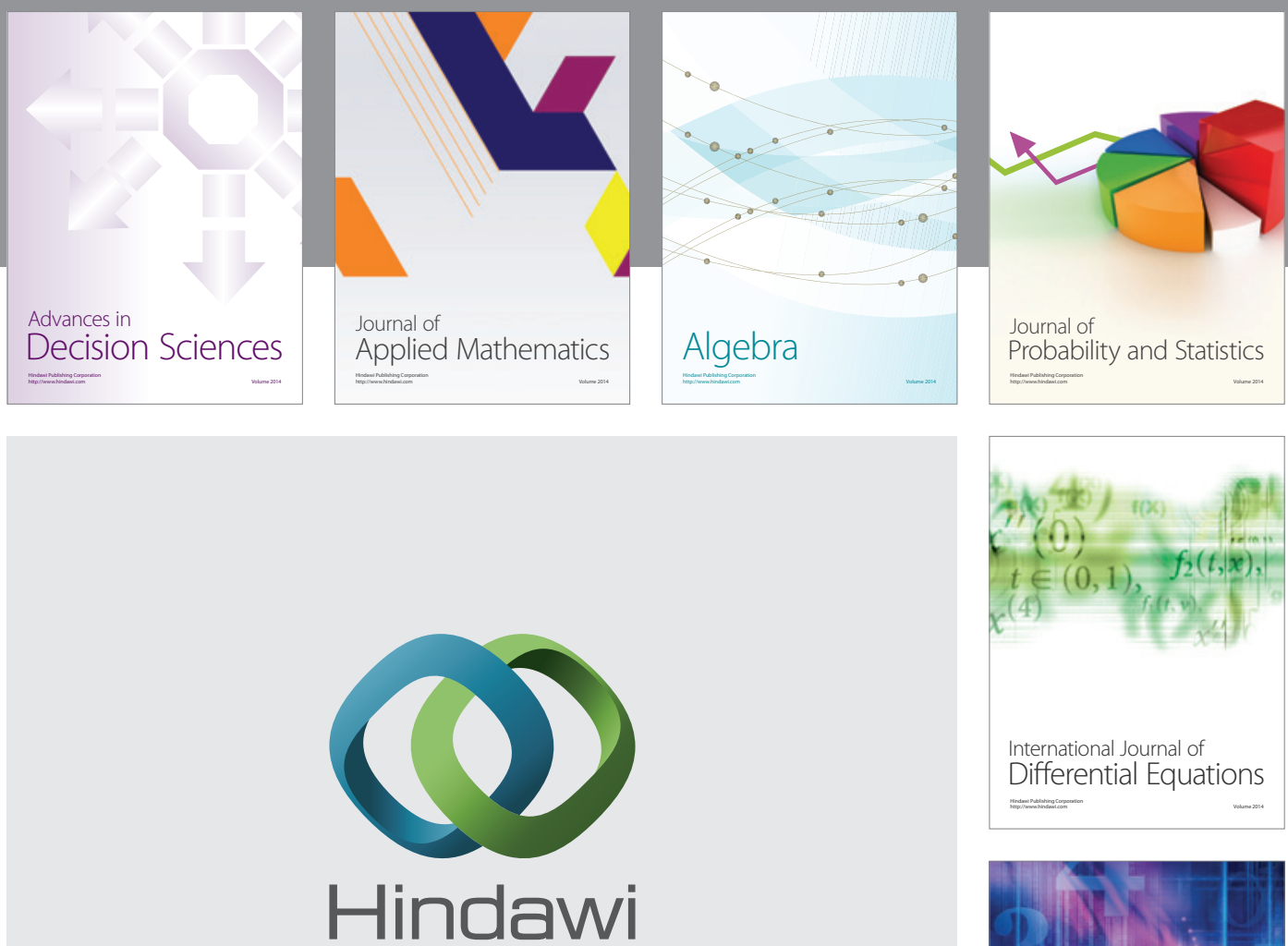

Submit your manuscripts at http://www.hindawi.com
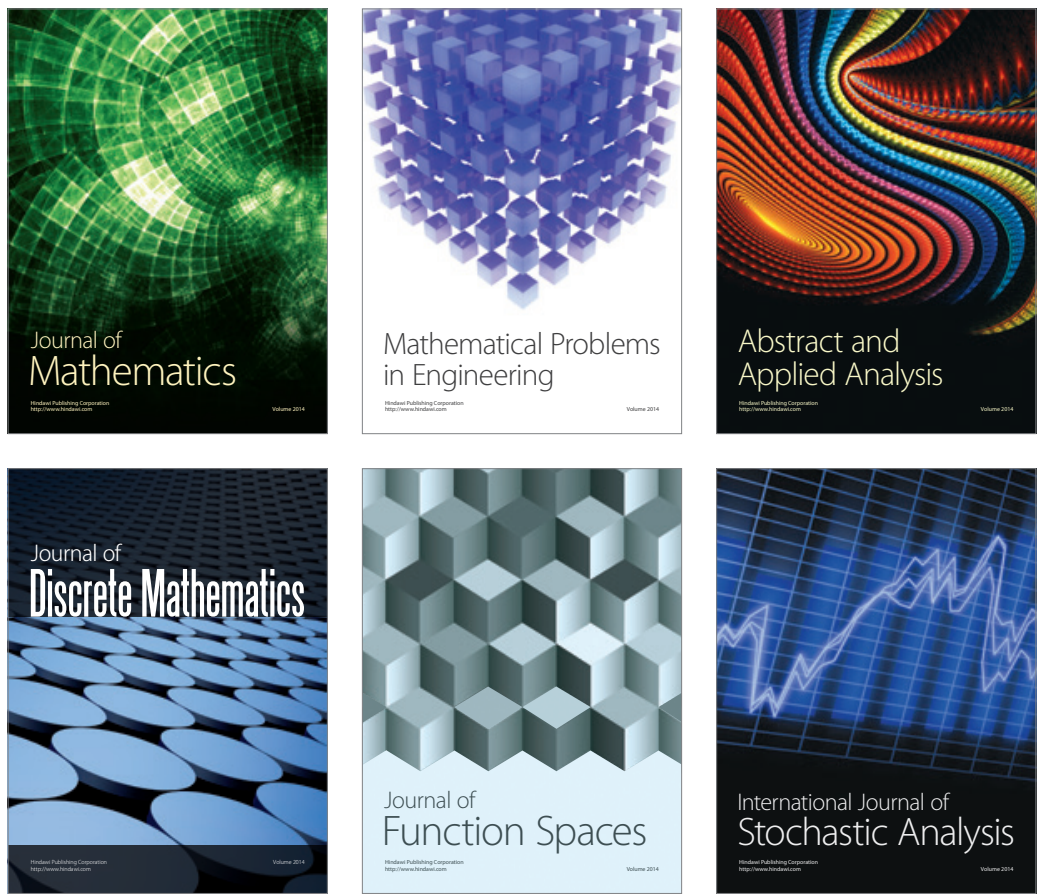

Journal of

Function Spaces

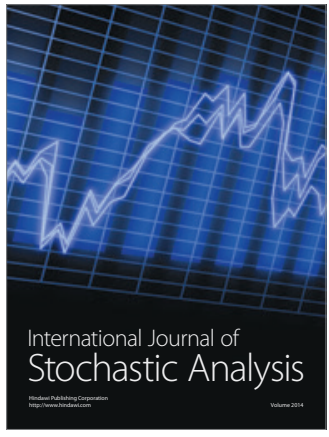

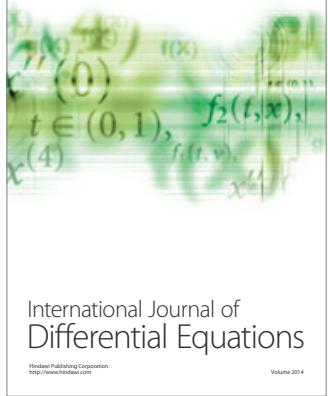
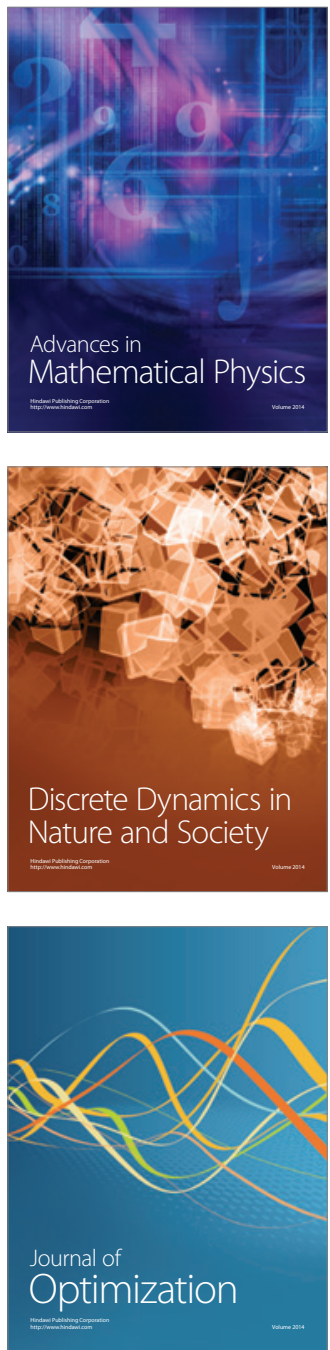